\section{Arrivoc

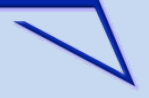

Archive for

Organic Chemistry
The Free Internet Journal

for Organic Chemistry
Paper

Arkivoc 2018, part iii, 45-61

\title{
Friedel-Crafts chemistry. Part 50. Convergent and diversity-oriented constructions of polycyclic quinolines via Friedel-Crafts and Beckmann ring enlargement approaches
}

\author{
Hassan A. K. Abd El-Aal \\ Chemistry Department, Faculty of Science, Assiut University, Assiut, 71516, Egypt \\ E-mail: hassankotb33@yahoo.com; hassankotb@aun.edu.eg
}

Received 06-23-2017

Accepted 10-22-2017

Published on line 12-03-2017

\section{Abstract}

Condensed heterocyclic systems containing N- \& S-medium-sized rings, in particular, thiazepine, thiazocine, and thiazonine systems are important substructures present in a large variety of biologically active natural products. Methods for the formation of thiazonines and higher ring systems, however, remain largely unknown. The research presented addresses the synthesis and characterization of new heterocyclic skeletons incorporating $\mathrm{N}$ \& S-medium-sized-rings fused to quinolines to form the targeted tetracyclic 1,4-thiazocines, 1,4-thiazonines and 1,4-thiazecines by Friedel-Crafts cycliacylation and Beckmann-rearrangement sequences. The ambient conditions, short-reaction times and easy work-up procedures make this synthetic strategy a better protocol for the synthesis of medium-sized heterocyclic rings bearing nitrogen and sulphur atoms.

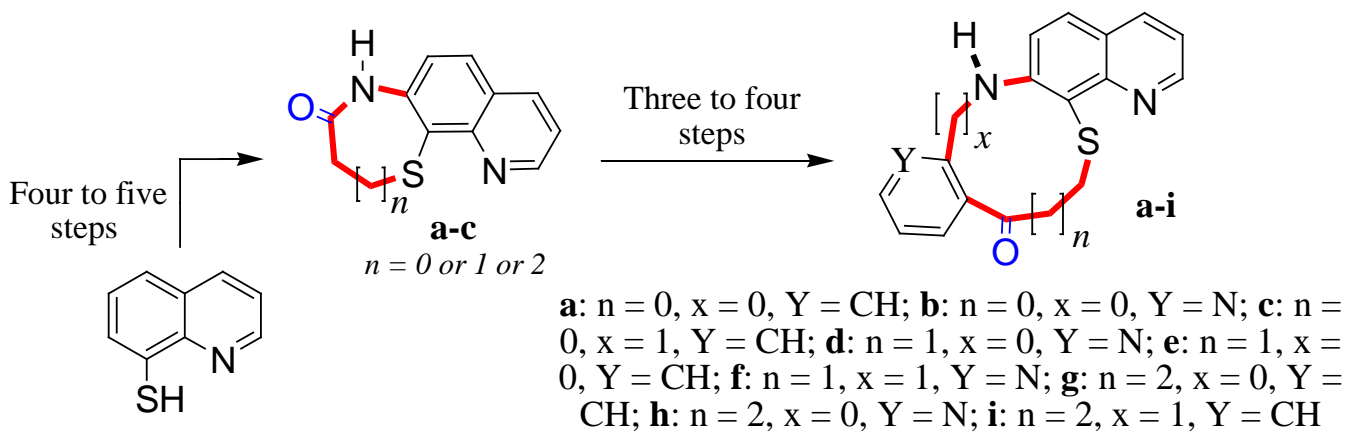

Keywords: Heteropolycycles, heterocyclic acids, 1,4-thiazocino[3,2-h]quinolinones, 1,4-thiazonino[3,2h]quinolinones 


\section{Introduction}

Condensed heterocycles containing $\mathrm{N}$ - and $\mathrm{S}$ - medium-sized rings, in particular thiazepine, thiazocine, and thiazonine systems, are receiving significant attention because of their presence in a wide range of natural products $^{1}$, and are often incorporated into biologically-active drugs and pharmaceuticals. ${ }^{2}$ Literature perusal of pharmacological studies of such moieties reveals that these compounds possess immense chemotherapeutic significance and are present as the core in a variety of drugs ${ }^{3}$ (Fig. 1), such as Prothioconazole-thiazocine, Diltiazem, Clotiapine, Omapatrilat, Promazine, Mesoridazine and Quetiapine, and exhibit a wide spectrum of pharmacological activities such as anticoagulant, ${ }^{4}$ antiarterisoclerotic, ${ }^{5}$ antihypertensive, ${ }^{6}$ antidepressant, ${ }^{7}$ antihistaminic, $^{8}$ anticonvulsant, ${ }^{9}$ antidopaminergic, ${ }^{10}$ tranquilizer, ${ }^{11}$ antidepressant, ${ }^{12}$ antihypertensive, ${ }^{13}$ calcium channel blocker, ${ }^{14}$ blood-platelet-aggregation inhibitors ${ }^{15}$ and antiallergic agents. ${ }^{16}$

A search for the applied methods for synthesis of medium-sized N,S-heterocyclic systems demonstrated that several established protocols are in practice, and comprehensive reviews of the syntheses and biological activities of various benzo-condensed 1,3-, 1,4-, 1,5-thiazepine and thiazocine regioisomers have been published. ${ }^{17-23}$ Formation of thiazonines and higher ring systems, however, remains unknown.

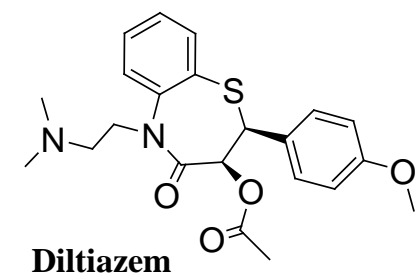

cis-(+)-[2-(2-Dimethylaminoethyl)-

5-(4-methoxyphenyl)-3oxo-6-thia-2-

azabicyclo[5.4.0]undeca-7,9,11trien-4-yl]ethanoate

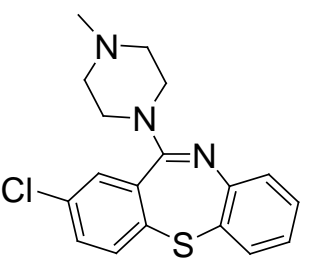

Clotiapine

8-chloro-6-(4-methylpiperazin-1yl)benzo[b][1,5]benzothiazepine

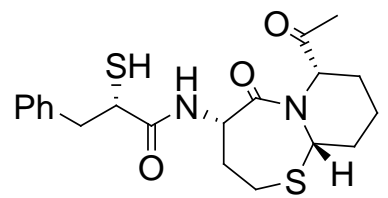

Omapatrilat

(4S,7S,10aS)-5-oxo-4-\{[(2S)-3-phenyl-2sulfanylpropanoyl]amino\}

-2,3,4,7,8,9,10,10aoctahydropyrido[6,1-b]

$[1,3]$ thiazepine-7-carboxylic acid

Figure 1. Fused medium-sized N,S-heterocycles-containing pharmaceuticals.

Most of the reported strategies for the synthesis of 1,4-thiazocine and higher ring frameworks are presented in a few select publications. For example, Yale et al. ${ }^{24}$ reported that dihydrodibenzo $[b, f] 1,4]$ thiazocine was obtained from derived $\alpha, \alpha$-dibromo-o-xylenes and 2-aminothiophenol in the presence of $\mathrm{NaHCO}_{3}$ in DMF solution. In 2004, Bates et al. ${ }^{25}$ reported a new strategy for the synthesis of several thiazocine-2-acetic acids from sulfoxide and sulfone analogues by ring-closing metathesis (RCM). They reported that a conjugate addition of allyl mercaptan to acrylate-containing olefinic intermediates, followed by RCM, provided the thiazocines in excellent yields.

Sashida et al. $^{26}$, on the other hand, reported interesting examples for the synthesis of eight-, nine-, and tenmembered rings of tetrahydro-1,2-thiazocines, hexahydro-1,2-thiazonines and 1,2-thiazecines through the 2,3sigmatropic shifts of $S$-imides of (Z)-2-vinylthiacycloalkanes. They disclosed that the ten-membered ring is generated directly during the treatment of chloramine $\mathrm{T}$ with 2-vinylthiacycloheptane. Manhas et al. ${ }^{27}$ synthesized several 6,7,8,9,10,11-hexahydro-10-methoxy-benzo[j][1,4]thiazonine-9,11-diones via enlargement (arynic condensation) by oxidation of the corresponding substituted S-lactam with $\mathrm{NaIO}_{4}$ in a water-isopropanol solution. Lu et $a .^{28}$ reported the formation of dibenzo[b,f][1,4]thiazocin-11-ones via the Pd-catalyzed carbonylation reactions of 2-(2-iodobenzylthio)benzenamines in low overall yields. 
Another isomerization-RCM strategy was carried out by van Otterlo et al. ${ }^{29}$ who reported that the synthesis of benzothiazocine dioxide from the corresponding sulfone was carried out in high yield. In an alternative strategy, Lilly et al. $^{30}$ reported a simple protocol for the synthesis of various benzo-1,4-thiazocines via intramolecular cyclization of acyclic-thioether substrates. Mukherjee et $a^{31}$ have applied the same intramolecular cyclization methodology to the synthesis of 2,3,4,5-tetrahydro-2Hbenzo[b][1,4]thiazocines by treatment of 3-(2-bromophenylthio)propan-1-amines (arenes) with lithium diisopropylamide (LDA). Federsel et al. ${ }^{32}$ reported the formation of a $\mathrm{N}$-formyl thiazocine series via the conversion of the thiazole and benzothiazole into the corresponding thiazolium salts, followed by ring expansion, which resulted in $N$-formyl thiazocine and benzothiazocine. Due to the wide range of biological, industrial and synthetic applications of these heterocyclic compounds, the development of a concise and efficient synthetic protocol for these moieties continues to challenge synthetic organic chemists.

In our previous works of this series, ${ }^{33,34}$ we described a straightforward synthesis of a novel series of $N$ carbocycles of various ring sizes via Friedel-Crafts ${ }^{35}$ cycliacylation reactions. In a continuation of these studies, the present research addresses the synthesis and characterization of new heterocyclic skeletons, incorporating N-\& S-medium-sized-rings nuclei fused to quinolines, to form the targeted tetracyclic 1,4-thiazocines, 1,4thiazonines and 1,4-thiazecines by applying the ring enlargement approaches of Friedel-Crafts and Beckmannrearrangement sequences.

\section{Results and Discussion}

Our synthetic route to the ( $N$-aryl- $N$-tosylamino)quinolin-8-ylthio)carboxylic acids precursors $10 a-i$ required for this work proceeded via consecutive steps starting from quinoline-8-thiol (1) as depicted in Scheme 1. Initial optimization studies were directed toward the ring-closure of acyclic precursors $4 a-c$, readily generated in a three-step sequence.

Synthesis was started by S-alkylation of quinoline-8-thiol (1), with different $\alpha-, \beta-$, and $\gamma$-bromoesters as alkylating agents, in the presence of $\mathrm{K}_{2} \mathrm{CO}_{3}$ in acetone to give ethyl (quinolin-8-ylthio)alkanoates (3a-c). The resulting esters were hydrolyzed by $\mathrm{NaOH}$ to yield the corresponding substituted 2-(quinolin-8-yl)sulfanyl) acids 4a-c.

Cyclization of the acids $4 a-c$ took place in the presence of polyphosphoric acid (PPA), producing the ketones thieno[3,2-h]quinolin-3(2H)-one (5a), 2,3-dihydrothiopyrano[3,2-h]quinolin-4-one $\quad(5 \mathbf{b}) \quad$ and 3,4dihydrothiepino[3,2-h]quinolin-5(2H)-one (5c) in moderate yields. Treatment of ketones $5 a-c$ with $\mathrm{NH}_{2} \mathrm{OH} . \mathrm{HCl}$ in $\mathrm{NaOH}$ solution gave the corresponding oximes 6 a-c. The resulting oximes underwent ring enlargement induced by heating with PPA at $110-120^{\circ} \mathrm{C}$ following Beckmann-rearrangement procedures ${ }^{36}$ to afford the corresponding cyclic amides 7a-c. These amides were hydrolyzed to the corresponding substituted (7aminoquinolin-8-ylthio)alkanoic acids $8 \mathrm{a}-\mathrm{c}$ with $\mathrm{NaOH}$ in refluxing $\mathrm{EtOH}$, which were subsequently converted to 7-bromoquinolin-8-ylthioalkanoic acids 9a-c by Sandmeyer reaction. ${ }^{37}$ Subsequent treatment of halo-acids 9a-c with various aromatic tosylated amines (PhNHTs or $\mathrm{N}$-tosylpyridin-2-amine or $\mathrm{TsNHCH}_{2} \mathrm{Ph}$ ) in the presence of $\mathrm{K}_{2} \mathrm{CO}_{3}$ in DMSO solution furnished (7-( $N$-phenyl- $N$-tosylamino)quinolin-8-ylthio)alkanoic acids (10a-i) in good overall yields. 


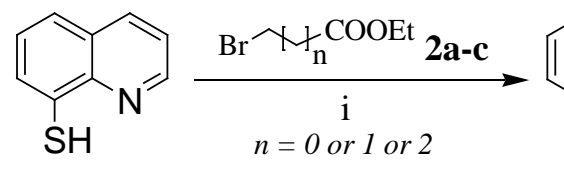

1

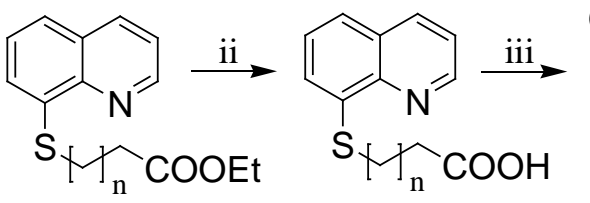

4a-c

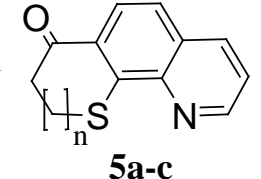

3a-c $\underset{9 a-c}{\text { Bromoacids }} \underset{\text { vii }}{\longleftarrow} \mathrm{H}_{2} \mathrm{~N}$

10a-i

a: $\mathrm{n}=0, \mathrm{Ar}=\mathrm{Ph} ; \mathbf{b}: \mathrm{n}=0, \mathrm{Ar}=2-$ Pyridyl; $\mathbf{c}: \mathrm{n}=0, \mathrm{Ar}=\mathrm{PhCH}_{2} ; \mathbf{d}: \mathrm{n}=$ $1, \mathrm{Ar}=\mathrm{Ph} ; \mathbf{e}: \mathrm{n}=1, \mathrm{Ar}=2-\mathrm{Pyridyl}$ f: $\mathrm{n}=1, \mathrm{Ar}=\mathrm{PhCH}_{2} ; \mathbf{g}: \mathrm{n}=2, \mathrm{Ar}=$ Ph; h: $n=2$, Ar =2-Pyridyl; $\mathbf{i}: \mathrm{n}=2$, $\mathrm{Ar}=\mathrm{PhCH}_{2}$

Scheme 1. Reagents and conditions: (i) $\mathrm{K}_{2} \mathrm{CO}_{3}$ /acetone, $15 \mathrm{~h}$, reflux, (ii) $\mathrm{NaOH}, 2-3 \mathrm{~h}$, reflux, (iii) polyphosphoric acid (PPA), 5h, $100-110^{\circ} \mathrm{C}$, (iv) $\mathrm{NH}_{2} \mathrm{OH}$. $\mathrm{HCl} / \mathrm{NaOH}, 1 \mathrm{~h}, 80-90^{\circ} \mathrm{C}$, (v) PPA, 5h, 110-120 ${ }^{\circ} \mathrm{C}$, (vi) EtOH/NaOH, $10 \mathrm{~h}$, reflux, (vii) $\mathrm{HCl} / \mathrm{NaNO}_{2} / \mathrm{H}_{2} \mathrm{O} / \mathrm{KBr}, 30 \mathrm{~min}, 100{ }^{\circ} \mathrm{C}$, (viii) Aromatic amines (PhNHTs or $\mathrm{N}$-tosylpyridin-2-amine or $\left.\mathrm{TsNHCH}_{2} \mathrm{Ph}\right), \mathrm{K}_{2} \mathrm{CO}_{3} / \mathrm{DMSO}, 120-130{ }^{\circ} \mathrm{C}, 10 \mathrm{~h}$.

Cycloacylations of acids $10 \mathrm{a}$-i were carried out in the presence of $\mathrm{AlCl}_{3} / \mathrm{CH}_{3} \mathrm{NO}_{2}$ or $\mathrm{P}_{2} \mathrm{O}_{5}$ or $\mathrm{p}$-toluenesulfonic acid (PTSA) catalysts providing a series of nine tetracyclic benzo- and pyrido- 1,4-thiazocinoquinolinones, 1,4thiazoninoquinolinones, 1,4-thiazecinoquinolinones and 1,4-thiazacyclododecano[3,2-h]quinolinones 11a-i (Scheme 2 and Table 1). The structures of all ketones were confirmed by both analytical and spectral data.

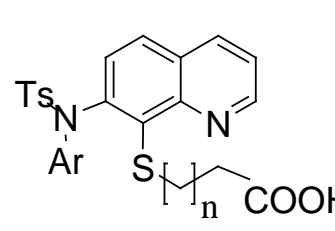

10a-i

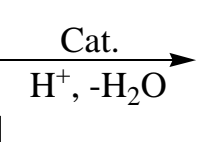

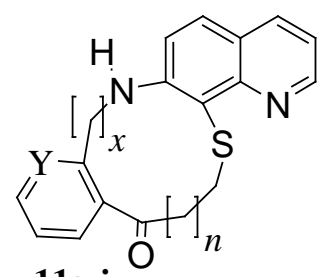

11a-i

a: $\mathrm{n}=0, \mathrm{x}=0, \mathrm{Y}=\mathrm{CH} ; \mathbf{b}: \mathrm{n}=0, \mathrm{x}=0, \mathrm{Y}$

$=\mathrm{N} ; \mathbf{c}: \mathrm{n}=0, \mathrm{x}=1, \mathrm{Y}=\mathrm{CH} ; \mathbf{d}: \mathrm{n}=1, \mathrm{x}=$

$0, \mathrm{Y}=\mathrm{N} ; \mathbf{e}: \mathrm{n}=1, \mathrm{x}=0, \mathrm{Y}=\mathrm{CH} ; \mathbf{f}: \mathrm{n}=1$,

$\mathrm{X}=1, \mathrm{Y}=\mathrm{N} ; \mathbf{g}: \mathrm{n}=2, \mathrm{x}=0, \mathrm{Y}=\mathrm{CH} ; \mathbf{h}: \mathrm{n}$

$=2, x=0, Y=N ; \mathbf{i}: n=2, x=1, Y=C H$

Scheme 2. Cycloacylations of acids 10a-i under Friedel-Crafts conditions.

The acylation mechanism accounting for the ring closure products involves the generation of acyl carbocations by loss of water or alcohol upon treatment with acidic catalysts. The resulting acyl carbocations underwent ring closure to form the fused tetracyclic quinolinones (11a-i) (Tables 1 and 2). The removal of the Ts-group takes place concurrently with the closure step of heterocyclic acids as noted in different examples in the literature. ${ }^{38}$ 
Table 1. Friedel-Crafts cycloacylations of heterocyclic acids 10a-c

\begin{tabular}{lll}
\hline Entry & \multicolumn{1}{c}{ Conditions } & \multicolumn{1}{c}{ Product (\%) } \\
\hline
\end{tabular}

asolated yield relative to substrate. ${ }^{b}$ With $\mathrm{AlCl}_{3} / \mathrm{CH}_{3} \mathrm{NO}_{2}$ catalyst reactant proportions were: acid (0.002 mole), $\mathrm{AlCl}_{3}$ (0.0024 mole), $\mathrm{CH}_{3} \mathrm{NO}_{2}\left(0.024\right.$ mole), solvent $(10 \mathrm{~mL})$. ${ }^{c}$ Dichloromethane. ${ }^{d}$ With $\mathrm{P}_{2} \mathrm{O}_{5}$ catalyst reactant proportions were: acid $(0.4 \mathrm{~g})$ and $\mathrm{P}_{2} \mathrm{O}_{5}(4 \mathrm{~g})$ in anhydrous toluene $(15 \mathrm{~mL})$. eWith PTSA catalyst reactant proportions were: acid ( $0.5 \mathrm{~g})$, PTSA ( $3 \mathrm{~g})$ and solvent $(10 \mathrm{~mL})$.

Table 2. Friedel-Crafts ring closures of acids $10 \mathrm{~d}-\mathbf{i}$

\begin{tabular}{lll} 
Entry & \multicolumn{1}{c}{ Conditions } & \multicolumn{1}{c}{ Product (\%) } \\
11d (85)
\end{tabular}




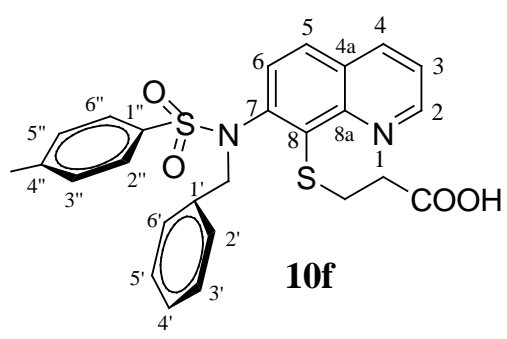

3-(7-(N-benzyl- $N$-tosylamino)quinolin8-ylthio)propanoic acid

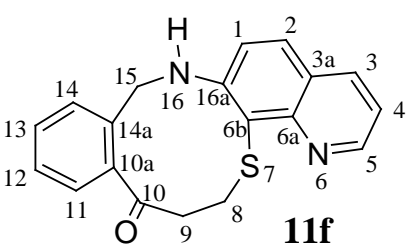

8,9,10,15-tetrahydro-16H-benzo[6,7][1,4] thiazecino[3,2-h]quinolin-10-one

Figure 2. Structures of tetracyclic $\mathbf{1 1 f}$ and its precursor heterocyclic acid $\mathbf{1 0 f .}$

The ${ }^{1} \mathrm{H}$ NMR data showed the obvious elucidation of the formation of condensed heteropolycycles. For example, the ${ }^{1} \mathrm{H}$ NMR spectrum for propanoic acid 10 displayed six signals in which $\mathrm{CH}_{3}$ protons showed as a singlet at $\delta 2.32 \mathrm{ppm}$, and two methylene groups appeared as two triplets at $\delta 2.76$ and $3.40 \mathrm{ppm}$. The fourth signal appeared as singlet at $\delta 5.13$ ppm for benzylic- $\mathrm{CH}_{2}$ protons, while aromatic protons gave sets of multiplets in the area of 7.00-8.83 ppm. The sixth singlet proton, which appeared at $\delta 10.70 \mathrm{ppm}$, was assigned to carboxylgroup protons. In comparison with acid 10f, tetracyclic thiazecino[3,2- $h$ ]quinolin-10-one 11f showed ${ }^{1} \mathrm{H}$ NMR chemical shifts in $\mathrm{CDCl}_{3}$ as a characteristic set of five signals. Three $\mathrm{CH}_{2}$-groups appeared as two triplets at $\delta 2.87$ \& $3.93 \mathrm{ppm}$, and a singlet at $\delta 4.52 \mathrm{ppm}$ was assigned to the down-field $\mathrm{CH}_{2}$ protons, respectively. The aromatic protons exhibited a complex set of signals at $\delta 6.89-8.72 \mathrm{ppm}$ and $\delta 9.95 \mathrm{ppm}$ for the NH group.

\section{Conclusions}

The development of a new, concise, and efficient protocol with broad applicability for the preparation of a range of tetracyclic, medium-sized $\mathrm{N}$ - \& S-heterocyclic rings fused to quinoline scaffolds using Friedel-Crafts ring closures of synthesized heterocyclic acids in the presence of $\mathrm{AlCl}_{3} / \mathrm{CH}_{3} \mathrm{NO}_{2}, \mathrm{P}_{2} \mathrm{O}_{5}$ and PTSA catalysts, and Beckmann rearrangements has been achieved. The ambient conditions, short reaction times and easy work-up procedures make this synthetic strategy a better protocol for the synthesis of medium-sized heterocyclic rings bearing nitrogen and sulphur atoms. The results have demonstrated the significance of a Friedel-Crafts ringclosure approach in the synthesis of heteropolycycles.

\section{Experimental Section}

General. All melting points were determined with a digital Gallenkamp capillary melting point apparatus and are uncorrected. Infrared spectra were measured on a Mattson 5000 FTIR spectrometer. ${ }^{1} \mathrm{H}$ NMR and ${ }^{13} \mathrm{C}$ NMR spectra were recorded on JEOL LA $400 \mathrm{MHz}$ FT-NMR (400 MHz for ${ }^{1} \mathrm{H}, 100 \mathrm{MHz}$ for ${ }^{13} \mathrm{C}$ ). Chemical shifts $(\delta)$ are reported in ppm downfield from TMS as the internal standard, and coupling constants are expressed as $J$ values in $\mathrm{Hz}$. Elemental analyses were carried out either by a Perkin-Elmer 2400 Series II instrument or by a microanalytical unit. Reactions were monitored by TLC using pre-coated silica plates ( $0.2 \mathrm{~mm}$, Kiesel 60, F254, E. Merck) and visualized with UV light. Flash column chromatography was performed on silica gel (230-400 mesh, E. Merck). 
General procedure for the synthesis of ethyl 2-(quinolin-8-ylthio)alkanoates (3a-c). A mixture of quinoline-8thiol 1 (3.2 g, $20 \mathrm{mmol}),\left(\alpha-, \beta\right.$ - or $\gamma$-) haloesters $2 \mathrm{a}$ or b or $\mathbf{c}(25 \mathrm{mmol})$ and anhydrous $\mathrm{K}_{2} \mathrm{CO}_{3}(7.0 \mathrm{~g}, 50 \mathrm{~mol})$ in dry acetone $(50 \mathrm{~mL})$ was refluxed in a water bath for $15 \mathrm{~h}$. On completion of the reaction, confirmed by TLC (20\% ethyl acetate/ $n$-hexane), the excess solvent was removed by distillation. The residue was diluted with water and finally extracted with ether $(3 \times 30 \mathrm{~mL})$. The combined organic layers were washed with water and dried over anhydrous $\mathrm{Na}_{2} \mathrm{SO}_{4}$. Evaporation of the solvent in vacuo afforded crude products. Purification of the crude esters by flash column chromatography (basic alumina, EtOAc/n-hexane, $1 / 3$ ) gave pure esters 3a-c. The yields, further purifications and spectral data are given in the following:

Ethyl 2-(quinolin-8-ylthio)acetate (3a). Yellow needles; $80 \%$; mp 70-2 ${ }^{\circ} \mathrm{C}$ (from n-hexane); $\mathrm{IR}\left(\mathrm{KBr}, \mathrm{v}, \mathrm{cm}^{-1}\right.$ ): $3065,2920,1745,1600,1575,1492,1440,1345,1234,1178,742 \mathrm{~cm}^{-1} ;{ }^{1} \mathrm{H} \mathrm{NMR}(400 \mathrm{MHz}, \mathrm{CDCl}, \mathrm{ppm}), \delta 1.17$ $\left(3 \mathrm{H}, \mathrm{t}, J 6.8\right.$, and $\left.7.6 \mathrm{~Hz}, \mathrm{CH}_{3}\right), 3.95\left(2 \mathrm{H}, \mathrm{s}, \mathrm{CH}_{2}\right), 4.12\left(2 \mathrm{H}, \mathrm{q}, J 7.2,7.2\right.$ and $\left.6.8 \mathrm{~Hz}, \mathrm{CH}_{2}\right), 7.37(1 \mathrm{H}, \mathrm{q}, J 4.8,3.6$ and $4.4 \mathrm{~Hz}), 7.60(1 \mathrm{H}, \mathrm{dd}, \mathrm{J} 6.0$, and $1.6 \mathrm{~Hz}), 7.81(1 \mathrm{H}, \mathrm{t}, J 7.6 \mathrm{~Hz}), 7.87(1 \mathrm{H}, \mathrm{d}, J 1.2 \mathrm{~Hz}), 8.09(1 \mathrm{H}, \mathrm{app} \mathrm{dt}, J 8.0 \mathrm{~Hz})$, $8.92(1 \mathrm{H}, \mathrm{dd}, J 2.0,2.8$ and $1.6 \mathrm{~Hz}) ;{ }^{13} \mathrm{C} \mathrm{NMR}\left(100 \mathrm{MHz}, \mathrm{CDCl}_{3}, \delta, \mathrm{ppm}\right): 14.1,36.4,61.5,122.1,125.5,126.6$, 127.4, 127.9, 130.9, 134.4, 144.8, 148.7, 169.3. Anal. Calcd. for $\mathrm{C}_{13} \mathrm{H}_{13} \mathrm{NO}_{2} \mathrm{~S}$ (247); C, 63.15; $\mathrm{H}, 5.26 ; \mathrm{N}, 5.66 ; \mathrm{S}$, 12.95. Found; C, 63.24; H, 5.22; N, 5.74; S, $12.80 \%$.

Ethyl 3-(quinolin-8-ylthio)propanoate (3b). Yellowish viscous oil; 88\%; $n_{D}^{25} 1.586$; IR (Film) $v_{\max } 3020,2968$, $1740,1590,1570,1460,1440,1330,1282,755 \mathrm{~cm}^{-1} ;{ }^{1} \mathrm{H}$ NMR $\left(400 \mathrm{MHz}, \mathrm{CDCl}_{3}, \mathrm{ppm}\right), \delta 1.32\left(3 \mathrm{H}, \mathrm{t}, J 7.2 \mathrm{~Hz}, \mathrm{CH}_{3}\right)$, $2.66\left(2 \mathrm{H}, \mathrm{t}, J 6.8\right.$ and $\left.6.4 \mathrm{~Hz}, \mathrm{CH}_{2}\right), 3.28\left(2 \mathrm{H}, \mathrm{t}, J 6.8\right.$ and $\left.7.2 \mathrm{~Hz}, \mathrm{CH}_{2}\right), 4.07\left(2 \mathrm{H}, \mathrm{q}, J 7.2 \mathrm{~Hz}, \mathrm{CH}_{2}\right), 7.36(1 \mathrm{H}, \mathrm{q}, J 4.8$ and $3.2 \mathrm{~Hz}), 7.59(1 \mathrm{H}, \mathrm{t}, J 6.0 \mathrm{~Hz}), 7.81(1 \mathrm{H}, \mathrm{t}, J 8.0 \mathrm{~Hz}), 7.86(1 \mathrm{H}, \mathrm{d}, J 2.0 \mathrm{~Hz}), 8.09(1 \mathrm{H}, \mathrm{td}, J 8.4 \mathrm{and} 1.2 \mathrm{~Hz}), 8.92$ $(1 \mathrm{H}, \mathrm{dd}, J 2.0$ and $1.6 \mathrm{~Hz}) ;{ }^{13} \mathrm{C} \mathrm{NMR}\left(100 \mathrm{MHz} \mathrm{CDCl}_{3}, \delta, \mathrm{ppm}\right): 14.1,28.7,34.5,60.6,122.1,125.5,126.6,127.5$, 127.9, 130.9, 134.4, 144.8, 148.7, 171.5. Anal. Calcd. for $\mathrm{C}_{14} \mathrm{H}_{15} \mathrm{NO}_{2} \mathrm{~S}(261) ; \mathrm{C}, 64.36 ; \mathrm{H}, 5.74 ; \mathrm{N}, 5.36 ; \mathrm{S}, 12.26$. Found; C, 64.34; H, 5.64; N, 5.50; S, $12.35 \%$.

Ethyl 4-(quinolin-8-ylthio)butanoate (3c). White crystals; $75 \%$; $\mathrm{mp} 95-7{ }^{\circ} \mathrm{C}$ [from petroleum ether $\left(60-80^{\circ} \mathrm{C}\right)$ ]; IR (KBr) $v_{\max } 3040,2945,1735,1580,1520,1485,1440,1370,1330,1185,742 \mathrm{~cm}^{-1}$; ${ }^{1} \mathrm{H} \mathrm{NMR}\left(400 \mathrm{MHz} \mathrm{CDCl}_{3}\right.$, ppm), $\delta 1.13\left(3 \mathrm{H}, \mathrm{t}, J 7.2\right.$ and $\left.6.8 \mathrm{~Hz}, \mathrm{CH}_{3}\right), 1.86\left(2 \mathrm{H}, \mathrm{sp}, J \mathrm{~J} .2,7.2,7.2\right.$ and $\left.7.6 \mathrm{~Hz}, \mathrm{CH}_{2}\right), 2.28(2 \mathrm{H}, \mathrm{t}, J 7.2$ and 7.6 $\left.\mathrm{Hz}, \mathrm{CH}_{2}\right), 3.09\left(2 \mathrm{H}, \mathrm{t}, J 7.2 \mathrm{~Hz}, \mathrm{CH}_{2}\right), 4.07\left(2 \mathrm{H}, \mathrm{q}, J 6.8,7.2 \mathrm{~Hz}, \mathrm{CH}_{2}\right), 7.37(1 \mathrm{H}, \mathrm{q}, J 3.2$ and $4.8 \mathrm{~Hz}), 7.56(1 \mathrm{H}, \mathrm{dd}, J$ 6.0 and $1.6 \mathrm{~Hz}), 7.81(1 \mathrm{H}, \mathrm{t}, J 8.0 \mathrm{~Hz}), 7.87(1 \mathrm{H}, \mathrm{d}, J 2.0 \mathrm{~Hz}), 8.09(1 \mathrm{H}, \mathrm{d}, J 7.6 \mathrm{~Hz}), 8.91(1 \mathrm{H}, \mathrm{dd}, J 2.0$ and $1.6 \mathrm{~Hz})$; ${ }^{13} \mathrm{C} \mathrm{NMR}\left(100 \mathrm{MHz}, \mathrm{CDCl}_{3}, \delta, \mathrm{ppm}\right): 14.1,24.8,32.4,33.2,60.6,122.1,125.5,126.7,127.5,127.9,130.9,134.4$, 144.8, 148.7, 173.0. Anal. Calcd. for $\mathrm{C}_{15} \mathrm{H}_{17} \mathrm{NO}_{2} \mathrm{~S}$ (275); C, 65.45; $\mathrm{H}, 6.18 ; \mathrm{N}, 5.09 ; \mathrm{S}, 11.63$. Found; $\mathrm{C}, 65.61 ; \mathrm{H}$, $6.25 ; \mathrm{N}, 5.12 ; \mathrm{S}, 11.82 \%$.

General procedure for the synthesis of propanoic acids (4a-c). A mixture of ester $3 \mathbf{a}$ or $\mathbf{b}$ or $\mathbf{c}(5 \mathrm{mmol})$ and excess $\mathrm{NaOH}$ solution ( $15 \mathrm{~mL}, 20 \%$ ) was stirred under reflux for 2-3 h. After cooling to room temperature, the clear solution was diluted with water $(30 \mathrm{~mL})$ and poured with occasionally stirring into an ice-cold diluted $\mathrm{HCl}$ solution (20 mL, 15\%). After standing for $8 \mathrm{~h}$ in a refrigerator, the crude acid was filtered off and dissolved with slight warming in a $\mathrm{NaHCO}_{3}$ solution (15 mL, $20 \%$ ), filtered and acidified with $\mathrm{HCl}$ solution (25 mL, 10\%). The product was filtered, washed and dried to afford crude acids $\mathbf{4 a}$, or $\mathbf{b}$ or $\mathbf{c}$. Purifications, yields and spectral data are presented in the following:

2-(Quinolin-8-ylthio)acetic acid (4a). White needles; 82\%; mp 108-10 ${ }^{\circ} \mathrm{C}$ (from methanol); IR $\left(\mathrm{KBr}, \mathrm{v}, \mathrm{cm}^{-1}\right): 3320$, 3085, 2950, 2620, 1718, 1585, 1450, 1435, 1330, 750. ${ }^{1} \mathrm{H} \mathrm{NMR}\left(400 \mathrm{MHz}, \mathrm{CDCl}_{3}, \mathrm{ppm}\right), \delta 3.97\left(2 \mathrm{H}, \mathrm{s}, \mathrm{CH}_{2}\right), 7.36$ $(1 \mathrm{H}, \mathrm{q}, J 4.8,3.2$ and $4.8 \mathrm{~Hz}), 7.58(1 \mathrm{H}, \mathrm{dd}, J 1.2,4.0$ and $1.6 \mathrm{~Hz}), 7.82(1 \mathrm{H}, \mathrm{t}, J 7.6 \mathrm{~Hz}), 7.87(1 \mathrm{H}, \mathrm{app} \mathrm{d}, J 2.0 \mathrm{~Hz})$, $8.07(1 \mathrm{H}, \mathrm{d}, J 8.0 \mathrm{~Hz}), 8.91(1 \mathrm{H}, \mathrm{dd}, J 2.0$ and $1.6 \mathrm{~Hz}), 10.35(1 \mathrm{H}, \mathrm{s}, \mathrm{COOH}) ;{ }^{13} \mathrm{C} \mathrm{NMR}\left(100 \mathrm{MHz}, \mathrm{CDCl}_{3}, \delta, \mathrm{ppm}\right)$ : $32.5,122.1,125.5,126.6,127.4,127.9,130.8,134.4,144.8,148.7,171.3$. Anal. Calcd. for $\mathrm{C}_{11} \mathrm{H}_{9} \mathrm{NO}_{2} \mathrm{~S}(219) ; \mathrm{C}$, $60.27 ; \mathrm{H}, 4.10 ; \mathrm{N}, 6.39 ; \mathrm{S}, 14.61$. Found; C, 60.41; H, 4.19; N, 6.43; S, 14.55\%. 
3-(Quinolin-8-ylthio)propanoic acid (4b). Pale yellow needles; 90\%; mp 122-4 ${ }^{\circ} \mathrm{C}$ (from benzene); IR (KBr, $\mathrm{V}, \mathrm{cm}^{-}$ $\left.{ }^{1}\right): 3035,2970,2560,1720,1600,1580,1460,1445,1335,1180,748 .{ }^{1} \mathrm{H} \mathrm{NMR}\left(400 \mathrm{MHz}, \mathrm{CDCl}_{3}, \mathrm{ppm}\right), \delta 2.69$ $\left(2 \mathrm{H}, \mathrm{t}, J 6.8 \mathrm{~Hz}, \mathrm{CH}_{2}\right), 3.25\left(2 \mathrm{H}, \mathrm{t}, J 6.8 \mathrm{~Hz}, \mathrm{CH}_{2}\right), 7.37(1 \mathrm{H}, \mathrm{app} \mathrm{q}, J 3.2$, and $4.8 \mathrm{~Hz}), 7.56(1 \mathrm{H}, \mathrm{dd}, J 1.6 \mathrm{and} 2.0 \mathrm{~Hz})$, $7.81(1 \mathrm{H}, \mathrm{t}, J 8.0 \mathrm{~Hz}), 7.86(1 \mathrm{H}$, app d, J $2.0 \mathrm{~Hz}), 8.09(1 \mathrm{H}, \mathrm{d}, J 8.4 \mathrm{~Hz}), 8.91(1 \mathrm{H}, \mathrm{dd}, J 2.0$ and $1.6 \mathrm{~Hz}), 11.25(1 \mathrm{H}$, s, $\mathrm{COOH}) ;{ }^{13} \mathrm{C} \mathrm{NMR}\left(100 \mathrm{MHz}, \mathrm{CDCl}_{3}, \delta, \mathrm{ppm}\right): 28.7,34.2,122.1,125.5,126.7,127.5,127.9,130.8,134.4,144.8$, 148.7, 175.2. Anal. Calcd. for $\mathrm{C}_{12} \mathrm{H}_{11} \mathrm{NO}_{2} \mathrm{~S}$ (233); C, 61.80; $\mathrm{H}, 4.72 ; \mathrm{N}, 6.00 ; \mathrm{S}, 13.73$. Found; C, 61.84; $\mathrm{H}, 4.65 ; \mathrm{N}$, $6.17 ;$ S, $13.58 \%$.

4-(Quinolin-8-ylthio)butanoic acid (4c). Creamy plates; 90\%; $\mathrm{mp} \mathrm{155-7}{ }^{\circ} \mathrm{C}$ (from methanol); IR $\left(\mathrm{KBr}, \mathrm{V}, \mathrm{cm}^{-1}\right)$ : $3030,2940,2560,1718,1610,1570,1455,1440,1380,1140,745 ;{ }^{1} \mathrm{H} \mathrm{NMR}\left(400 \mathrm{MHz}, \mathrm{CDCl}_{3}, \mathrm{ppm}\right), \delta 1.85(2 \mathrm{H}$, $\mathrm{sp}, J 7.2$, and $\left.7.6 \mathrm{~Hz}, \mathrm{CH}_{2}\right), 2.41\left(2 \mathrm{H}, \mathrm{t}, J 7.2 \mathrm{~Hz}, \mathrm{CH}_{2}\right), 3.08\left(2 \mathrm{H}, \mathrm{t}, J 7.6 \mathrm{~Hz}, \mathrm{CH}_{2}\right), 7.37(1 \mathrm{H}$, app q, J 3.2, and $4.8 \mathrm{~Hz})$, $7.56(1 \mathrm{H}, \mathrm{dd}, J 1.6$ and $2.0 \mathrm{~Hz}), 7.81(1 \mathrm{H}, \mathrm{t}, J 8.0 \mathrm{~Hz}), 7.86(1 \mathrm{H}$, app d, J $1.2 \mathrm{~Hz}), 8.09(1 \mathrm{H}, \mathrm{d}, J 7.6 \mathrm{~Hz}), 8.91(1 \mathrm{H}$, dd, $J 2.0$ and $1.6 \mathrm{~Hz}), 10.57(1 \mathrm{H}, \mathrm{s}, \mathrm{COOH}) ;{ }^{13} \mathrm{C} \mathrm{NMR}\left(100 \mathrm{MHz}, \mathrm{CDCl}_{3}, \delta, \mathrm{ppm}\right): 24.8,32.9,33.2,122.1,125.5$, 126.6, 127.4, 127.9, 130.8, 134.4, 144.8, 148.7, 177.6. Anal. Calcd. for $\mathrm{C}_{13} \mathrm{H}_{13} \mathrm{NO}_{2} \mathrm{~S}(247) ; \mathrm{C}, 63.15 ; \mathrm{H}, 5.26 ; \mathrm{N}$, 5.66; S, 12.95. Found; C, 63.19; H, 5.32; N, 5.49; S, $13.05 \%$.

General procedure for cyclization of propanoic acids (4a-c). A mixture of acid 4 a or b or c (5 mmol) and freshly prepared PPA (15 g) was heated on an oil bath and kept at temperature $100-110{ }^{\circ} \mathrm{C}$ for $5 \mathrm{~h}$. Afterwards, the flask was cooled to room temperature and basified by addition of $\mathrm{NaHCO}_{3}$ solution $(25 \mathrm{~mL}, 30 \%)$. The residue was extracted with ether $(3 \times 20 \mathrm{~mL})$ and the combined organic phases were washed with water, dried over anhydrous $\mathrm{Na}_{2} \mathrm{SO}_{4}$ and the solvent was evaporated in vacuo to give the crude ketones $\mathbf{5 a - c .}$

Thieno[3,2-h]quinolin-3(2H)-one (5a). Yellow plates; 84\%; mp 173-5 ${ }^{\circ} \mathrm{C}$ (ethanol); IR ( $\left.\mathrm{KBr}, \mathrm{v}, \mathrm{cm}^{-1}\right): 3050,2930$, $1692,1580,1470,1440,1385,1280,1075,749 .{ }^{1} \mathrm{H} \mathrm{NMR}\left(400 \mathrm{MHz}, \mathrm{CDCl}_{3}, \mathrm{ppm}\right), \delta 4.73\left(2 \mathrm{H}, \mathrm{s}, \mathrm{CH}_{2}\right), 7.40(1 \mathrm{H}, \mathrm{q}$, J 3.6 and $3.2 \mathrm{~Hz}), 7.81(1 \mathrm{H}, \mathrm{d}, J 10.4 \mathrm{~Hz}), 8.28(1 \mathrm{H}, \mathrm{td}, J 1.6,6.4$ and $1.6 \mathrm{~Hz}), 8.49(1 \mathrm{H}$, app dd, J $1.6 \mathrm{~Hz}), 8.98(1 \mathrm{H}$, dd, $J 2.0$ and $1.6 \mathrm{~Hz}) ;{ }^{13} \mathrm{C} \mathrm{NMR}\left(100 \mathrm{MHz} \mathrm{CDCl}_{3}, \delta, \mathrm{ppm}\right): 36.8,122.1,126.6,127.8,127.9,130.8,134.4,135.5$, 144.8, 150.8, 194.4. Anal. Calcd. for $\mathrm{C}_{11} \mathrm{H}_{7} \operatorname{NOS}(201) ; \mathrm{C}, 65.67 ; \mathrm{H}, 3.48 ; \mathrm{N}, 6.96 ; \mathrm{S}, 15.92$. Found; C, 65.60; $\mathrm{H}, 3.52$; N, 6.87; S, 15.95\%.

2,3-Dihydrothiopyrano[3,2-h]quinolin-4-one (5b). Creamy plates; 80\%; mp 152-4 ${ }^{\circ} \mathrm{C}$ (from methanol); IR (KBr, $\left.v, \mathrm{~cm}^{-1}\right): 3045,2962,1697,1583,1475,1440,1380,1340,1120,760 .{ }^{1} \mathrm{H} \mathrm{NMR}\left(400 \mathrm{MHz}, \mathrm{CDCl}_{3}, \mathrm{ppm}\right), \delta 2.79(2 \mathrm{H}$, $\mathrm{sp}, J 4.8,4.84 .4$ and $\left.4.8 \mathrm{~Hz}, \mathrm{CH}_{2}\right), 4.01\left(2 \mathrm{H}\right.$, app t, J 0.8, 3.6 and $\left.36 \mathrm{~Hz}, \mathrm{CH}_{2}\right), 7.40(1 \mathrm{H}, \mathrm{q}, J 3.6,3.2$ and $3.6 \mathrm{~Hz})$, $7.67(1 \mathrm{H}, \mathrm{d}, J 8.8 \mathrm{~Hz}), 7.98(1 \mathrm{H}, \mathrm{td}, J 1.6,1.6,1.2$ and $1.6 \mathrm{~Hz}), 8.48(1 \mathrm{H}, \mathrm{app} \mathrm{dd}, J 1.2 \mathrm{~Hz}), 8.98(1 \mathrm{H}, \mathrm{dd}, J 2.0$ and $1.6 \mathrm{~Hz}) ;{ }^{13} \mathrm{C}$ NMR $\left(100 \mathrm{MHz}, \mathrm{CDCl}_{3}, \delta, \mathrm{ppm}\right): 26.8,39.3,122.1,126.6,127.8,127.9,129.7,130.9,134.4,144.8$, 148.7, 192.1. Anal. Calcd. for $\mathrm{C}_{12} \mathrm{H}_{9} \mathrm{NOS}$ (215); C, 66.97; H, 4.18; N, 6.51; S, 14.88. Found; C, 67.07; H, 4.02; N, $6.58 ;$ S, $14.74 \%$.

3,4-Dihydrothiepino[3,2-h] quinolin-5(2H)-one (5c). Yellow needles; 79\%; mp 120-2 ${ }^{\circ} \mathrm{C}$ (from n-hexane); IR (KBr, $\left.\mathrm{v}, \mathrm{cm}^{-1}\right)$ : 3030, 2955, 1695, 1580, 1470, 1435, 1384, 1345, 1090, 754. ${ }^{1} \mathrm{H} \mathrm{NMR}\left(400 \mathrm{MHz}, \mathrm{CDCl}_{3}, \mathrm{ppm}\right), \delta 2.02(2 \mathrm{H}$, quin, J 4.0, 2.0, 2.4, 4.4 and $\left.4.0 \mathrm{~Hz}, \mathrm{CH}_{2}\right), 2.71\left(2 \mathrm{H}, \mathrm{t}, J 4.4\right.$ and $\left.3.6 \mathrm{~Hz}, \mathrm{CH}_{2}\right), 3.75\left(2 \mathrm{H}, \mathrm{t}, J 4.4 \mathrm{and} 3.6 \mathrm{~Hz}, \mathrm{CH}_{2}\right)$, $7.40(1 \mathrm{H}, \mathrm{q}, J 3.6,3.2$ and $3.6 \mathrm{~Hz}), 7.74(1 \mathrm{H}, \mathrm{d}, J 8.8 \mathrm{~Hz}), 7.99(1 \mathrm{H}, \mathrm{td}, J 1.6,2.4$ and $1.6 \mathrm{~Hz}), 8.40(1 \mathrm{H}, \mathrm{app} \mathrm{dd}, J$ $1.2 \mathrm{~Hz}), 8.98(1 \mathrm{H}$, app dd, $J 1.6$ and $3.6 \mathrm{~Hz}) ;{ }^{13} \mathrm{C} \mathrm{NMR}\left(100 \mathrm{MHz}, \mathrm{CDCl}_{3}, \delta, \mathrm{ppm}\right): 26.0,30.0,38.7,122.1,126.6$, $127.8,127.9,129.7,130.8,134.4,144.81,148.7,199.2$. Anal. Calcd. for $\mathrm{C}_{13} \mathrm{H}_{11} \mathrm{NOS}(229) ; \mathrm{C}, 68.12 ; \mathrm{H}, 4.80 ; \mathrm{N}$, 6.11; S, 13.97. Found; C, 68.20; H, 4.71; N, 6.16; S, $13.82 \%$.

General Procedure for the synthesis of cyclic amides (7a-c). The title compounds were obtained by applying Beckmann rearrangement of oximes 6a-c. Hence, these skeletons were obtained in a series of two steps. A summary of the steps is given in the following: 
Method (i) A solution of $\mathrm{NH}_{2} \mathrm{OH}$. $\mathrm{HCl}(0.68 \mathrm{~g}, 10 \mathrm{mmol})$ in water $(4 \mathrm{~mL})$ was added dropwise with stirring to an ice-cold solution of ketones $5 \mathbf{a}$ or $\mathbf{b}$ or $\mathbf{c}(8 \mathrm{mmol})$ in ethanol $(20 \mathrm{~mL})$. Lastly, a solution of $\mathrm{NaOH}(4 \mathrm{~mL}, 4 \mathrm{~N}) \mathrm{was}$ added gradually with efficient stirring during $5 \mathrm{~min}$. The cooling bath was then removed and the reaction mixture was left to stir for $30 \mathrm{~min}$ at room temperature, and finally heated in a steam bath at $80-90{ }^{\circ} \mathrm{C}$ for $1 \mathrm{~h}$. After cooling to room temperature, the reaction mixture was quenched by adding $50 \mathrm{~mL}$ of water. The resulting mixture was extracted with AcOEt $(3 \times 30 \mathrm{~mL})$ and the combined organic layers were washed with $\mathrm{HCl}(2 \times 30 \mathrm{~mL}$, $5 \%)$ and water. The organic extracts were dried over anhydrous $\mathrm{Na}_{2} \mathrm{SO}_{4}$, filtered, and evaporated to afford the crude oxime. The residue was purified by flash column chromatography (basic alumina, EtOAc/n-hexane, 1/1) resulting in the pure oximes $6 \mathbf{6}-\mathbf{c}$. The yields and spectral data are given in the following:

Thieno[3,2-h]quinolin-3(2H)-one oxime (6a). White crystal, 88\%; mp 98-100 ${ }^{\circ} \mathrm{C}$ (from ethanol); IR (KBr) 3175, 3160, 2940, 1664, 1476, 1380; ${ }^{1} \mathrm{H}$ NMR (400 MHz, CDCl, $\left.\mathrm{ppm}\right), \delta 4.89\left(2 \mathrm{H}, \mathrm{s}, \mathrm{CH}_{2}\right), 7.42(1 \mathrm{H}, \mathrm{q}, J 3.6 \mathrm{~Hz}), 7.87$ $(1 \mathrm{H}, \mathrm{d}, J 3.6 \mathrm{~Hz}), 8.24(1 \mathrm{H}$, app dd, J $1.6 \mathrm{~Hz}), 8.33(1 \mathrm{H}, \mathrm{td}, J 1.6,1.6,3.6$ and $2.0 \mathrm{~Hz}), 8.93(1 \mathrm{H}, \mathrm{dd}, J 1.6 \mathrm{and} 3.2$ $\mathrm{Hz}), 10.42(1 \mathrm{H}, \mathrm{s}, \mathrm{N}-\mathrm{OH}) ;{ }^{13} \mathrm{C} \mathrm{NMR}\left(100 \mathrm{MHz}, \mathrm{CDCl}_{3}, \delta, \mathrm{ppm}\right): 36.8,122.1,126.6,127.8,127.9,130.6,130.9,134.4$, 144.8, 150.8, 153.8. Anal. Calcd. for $\mathrm{C}_{11} \mathrm{H}_{8} \mathrm{~N}_{2} \mathrm{OS}$ (216); C, 61.11; H, 3.70; N, 12.96; S, 14.81. Found; C, 61.24; $\mathrm{H}$, $3.85 ; \mathrm{N}, 12.82 ; \mathrm{S}, 14.74 \%$.

2,3-Dihydrothiopyrano[3,2-h]quinolin-4-one oxime (6b). Pale yellow plates, $90 \%$; $\mathrm{mp} 111-13^{\circ} \mathrm{C}$ (from ethanol); IR (KBr) 3250, 3180, 2960, 1595, 1485, 1267; $\left.{ }^{1} \mathrm{H} \mathrm{NMR} \mathrm{(400} \mathrm{MHz,} \mathrm{CDCl}, \mathrm{ppm}\right), \delta 2.84$ (2H, app quin, J 4.0, 3.6, 1.2, 2.0, 2.8 and $\left.4.8 \mathrm{~Hz}, \mathrm{CH}_{2}\right), 3.98\left(2 \mathrm{H}, \mathrm{t}, J 4.8\right.$ and $\left.4.4 \mathrm{~Hz}, \mathrm{CH}_{2}\right), 7.42(1 \mathrm{H}, \mathrm{q}, J 4.8,3.2$ and $4.8 \mathrm{~Hz}), 7.64(1 \mathrm{H}, \mathrm{d}, J$ $7.2 \mathrm{~Hz}), 8.25(1 \mathrm{H}$, app sp, J 1.6, 1.6, 2.4 and $2.0 \mathrm{~Hz}), 8.40(1 \mathrm{H}, \mathrm{q}, J 2.0,2.8$ and $1.6 \mathrm{~Hz}), 8.93(1 \mathrm{H}$, app dd, $J 2.0,2.8$ and 1.6 Hz), $10.73(1 \mathrm{H}, \mathrm{s}, \mathrm{N}-\mathrm{OH}) ;{ }^{13} \mathrm{C} \mathrm{NMR}\left(100 \mathrm{MHz}, \mathrm{CDCl}_{3}, \delta, \mathrm{ppm}\right): 30.0,43.6,122.1,126.7,127.8,127.9,130.6$, $130.8,134.4,144.8,148.7,153.8$. Anal. Calcd. for $\mathrm{C}_{12} \mathrm{H}_{10} \mathrm{~N}_{2} \mathrm{OS}(230) ; \mathrm{C}, 62.60 ; \mathrm{H}, 4.34 ; \mathrm{N}, 12.17 ; \mathrm{S}, 13.91$. Found; C, 62.46; H, 4.52; N, 12.04; S, $14.07 \%$.

3,4-Dihydrothiepino[3,2-h]quinolin-5(2H)-one oxime (6c). White plates, 90\%; $\mathrm{mp} 160-2{ }^{\circ} \mathrm{C}$ (from methanol); IR ( $\mathrm{KBr}) 3220,3155,2927,1580,1490,1365 ;{ }^{1} \mathrm{H} \mathrm{NMR}\left(400 \mathrm{MHz}, \mathrm{CDCl}_{3}, \mathrm{ppm}\right), \delta 1.90$ (2H, app quin, J 4.0, 2.8, 1.6, 1.6, 2.8, 3.2 and $\left.3.2 \mathrm{~Hz}, \mathrm{CH}_{2}\right), 2.91\left(2 \mathrm{H}\right.$, app t, J 2.0, 2.4, 2.4 and $\left.2.0 \mathrm{~Hz}, \mathrm{CH}_{2}\right), 3.74\left(2 \mathrm{H}, \mathrm{t}, J 4.0\right.$ and $\left.4.8 \mathrm{~Hz}, \mathrm{CH}_{2}\right)$, $7.42(1 \mathrm{H}, \mathrm{q}, J 4.8,3.2$ and $4.8 \mathrm{~Hz}), 7.63(1 \mathrm{H}, \mathrm{d}, J 8.8 \mathrm{~Hz}), 8.24(1 \mathrm{H}$, app t, J 7.2 and $1.6 \mathrm{~Hz}), 8.33(1 \mathrm{H}$, quin, $J 1.6$, 1.6, 4.8 and $2.0 \mathrm{~Hz}), 8.93(1 \mathrm{H}$, app dd, J 1.6, 3.2 and $1.6 \mathrm{~Hz}), 10.20(1 \mathrm{H}, \mathrm{s}, \mathrm{N}-\mathrm{OH}) ;{ }^{13} \mathrm{C} \mathrm{NMR}(100 \mathrm{MHz}, \mathrm{CDCl}, \delta$, ppm): 26.0, 28.3, 30.0, 122.1, 126.6, 127.8, 127.9, 130.6, 130.8, 134.4, 144.8, 148.7, 153.8. Anal. Calcd. for $\mathrm{C}_{13} \mathrm{H}_{12} \mathrm{~N}_{2} \mathrm{OS}$ (244); C, 63.93; $\mathrm{H}, 4.91 ; \mathrm{N}, 11.47 ; \mathrm{S}, 13.11$. Found; C, 63.95; H, 4.80; N, 11.55; S, $13.15 \%$.

Method (ii) A mixture of oximes $6 \mathbf{a}$ or $\mathbf{b}$ or $\mathbf{c}(10 \mathrm{mmol})$ and polyphosphoric acid $(20.0 \mathrm{~g})$ was stirred at $110-$ $120{ }^{\circ} \mathrm{C}$ for $5 \mathrm{~h}$. The reaction mixture was cooled to room temperature, diluted with water $(50 \mathrm{~mL})$, saturated with $\mathrm{NaHCO}_{3}$ solution $(50 \mathrm{~mL}, 40 \%)$ and then left to stand overnight. The separated solid was filtered and washed with water to afford the respective cyclic amide. Recrystallization from ethanol gave the following:

$\mathbf{2 H}$-[1,4]Thiazino[3,2-h]quinolin-3(4H)-one (7a). White crystal, 76\%; mp 96-8 ${ }^{\circ} \mathrm{C}$ (from acetone); IR (KBr) 3460 , 3055, 2920, 1682, 1485; ${ }^{1} \mathrm{H}$ NMR (400 MHz, CDCl, $\left.\mathrm{ppm}\right), \delta 4.07\left(2 \mathrm{H}, \mathrm{s}, \mathrm{CH}_{2}\right), 7.32(2 \mathrm{H}, \mathrm{q}, J 4.8,4.0$ and $3.6 \mathrm{~Hz})$, $7.90(1 \mathrm{H}, \mathrm{dt}, J 1.6 \mathrm{~Hz}), 7.92(1 \mathrm{H}, \mathrm{d}, J 1.6 \mathrm{~Hz}), 8.11(1 \mathrm{H}, \mathrm{q}, J 1.6,7.2$ and $2.0 \mathrm{~Hz}), 8.76(1 \mathrm{H}, \mathrm{dd}, J 1.6,3.2 \mathrm{and} 1.6 \mathrm{~Hz})$, 10.04 (1H, s, NH); ${ }^{13} \mathrm{CNMR}\left(100 \mathrm{MHz} \mathrm{CDCl}_{3}, \delta, \mathrm{ppm}\right): 28.8,117.3,122.1,126.6,127.9,130.8,134.4,140.7,144.8$, 148.7, 165.0. Anal. Calcd. for $\mathrm{C}_{11} \mathrm{H}_{8} \mathrm{~N}_{2} \mathrm{OS}$ (216); C, 61.11; H, 3.70; N, 12.96; S, 14.81. Found; C, 61.19; H, 3.68; N, $12.82 ;$ S, $14.95 \%$.

2,3-Dihydro-[1,4]thiazepino[3,2-h]quinolin-4(5H)-one (7b). White crystal, 74\%; mp 117-9 ${ }^{\circ} \mathrm{C}$ (from methanol); IR (KBr) 3440, 3070, 2930, 1690, 1515, 1432; ${ }^{1} \mathrm{H}$ NMR (400 MHz, CDCl 3 , ppm), $\delta 2.71$ (2H, app t, J 5.2, 4.8 and $\left.4.4 \mathrm{~Hz}, \mathrm{CH}_{2}\right), 3.88\left(2 \mathrm{H}, \mathrm{t}, J 5.6\right.$ and $\left.5.2 \mathrm{~Hz}, \mathrm{CH}_{2}\right), 7.32(1 \mathrm{H}, \mathrm{q}, J 4.4,4.0$ and $4.8 \mathrm{~Hz}), 7.56(1 \mathrm{H}, \mathrm{d}, J 8.8 \mathrm{~Hz}), 7.89(1 \mathrm{H}$, quin, J 2.0, 1.6, 7.2 and 1.2 Hz), $8.10(1 \mathrm{H}, \mathrm{dd}, J 1.6$, and $7.6 \mathrm{~Hz}), 8.76(1 \mathrm{H}, \mathrm{dd}, J 2.0,2.8$ and $1.6 \mathrm{~Hz}), 10.51(1 \mathrm{H}, \mathrm{s}$, $\mathrm{NH}) ;{ }^{13} \mathrm{C} \mathrm{NMR}\left(100 \mathrm{MHz}, \mathrm{CDCl}_{3}, \delta, \mathrm{ppm}\right): 30.0,35.6,117.3,122.1,126.6,127.9,130.8,134.4,140.7,144.8,148.7$, 
170.6. Anal. Calcd. for $\mathrm{C}_{12} \mathrm{H}_{10} \mathrm{~N}_{2} \mathrm{OS}$ (230); C, 62.60; H, 4.34; N, 12.17; S, 13.91. Found; C, 62.60; H, 4.47; N, 12.20; $\mathrm{S}, 13.82 \%$.

3,4-Dihydro-2H-[1,4]thiazocino[3,2-h]quinolin-5(6H)-one (7c). Brown crystal, 80\%; mp 142-4 ${ }^{\circ} \mathrm{C}$ (from AcOEt); IR (KBr) 3420, 3050, 2957, 1700, 1520, 1450; ${ }^{1} \mathrm{H}$ NMR (400 MHz, CDCl, $\mathrm{ppm}$ ), $\delta 1.90$ (2H, quin, J 4.8, 2.4, 2.0, 3.2 and $\left.1.2 \mathrm{~Hz}, \mathrm{CH}_{2}\right), 2.47\left(2 \mathrm{H}, \mathrm{sp}, J 1.6,3.2,2.4\right.$ and $\left.1.6 \mathrm{~Hz}, \mathrm{CH}_{2}\right), 3.48\left(2 \mathrm{H}, \mathrm{t}, J 4.4\right.$ and $\left.4.8 \mathrm{~Hz}, \mathrm{CH}_{2}\right), 7.32-7.36(2 \mathrm{H}$, m), $7.89(1 \mathrm{H}, \mathrm{dt}, J 2.0,6.4,2.4$ and $1.2 \mathrm{~Hz}), 8.28(1 \mathrm{H}, \mathrm{app} \mathrm{dd}, J 1.6,7.2$ and $2.0 \mathrm{~Hz}$ ), $8.76(1 \mathrm{H}, \mathrm{dd}, J 2.8$ and 1.6 $\mathrm{Hz}$ ), $9.83(1 \mathrm{H}, \mathrm{s}, \mathrm{NH}) ;{ }^{13} \mathrm{C} \mathrm{NMR}\left(100 \mathrm{MHz} \mathrm{CDCl}_{3}, \delta, \mathrm{ppm}\right): 26.0,33.2$ (2C), 117.3, 122.1, 126.6, 127.9, 130.8, 134.4, 140.7, 144.8, 148.7, 174.0. Anal. Calcd. for $\mathrm{C}_{13} \mathrm{H}_{12} \mathrm{~N}_{2} \mathrm{OS}$ (244); C, 63.93; H, 4.91; N, 11.47; S, 13.11. Found; C, $64.14 ; \mathrm{H}, 4.84 ; \mathrm{N}, 11.37 ; \mathrm{S}, 13.15 \%$.

General Procedure for hydrolysis of cyclic amides 7a-c to aminoacids (8a-c). To a solution of cyclic amide $4 a$ or $\mathbf{b}$ or $\mathbf{c}(20 \mathrm{mmol})$ in ethanol $(30 \mathrm{~mL})$ was added $\mathrm{NaOH}$ solution $(10 \mathrm{~N}, 3.5 \mathrm{~mL})$; the resulting mixture was stirred under reflux for $10 \mathrm{~h}$. The excess alcohol was removed by distillation and the residue was diluted with water (50 $\mathrm{mL}$ ). The resulting solution was filtered, and the filtrate was cooled to room temperature and adjusted to $\mathrm{pH} 6$ 7 with $\mathrm{HCl}$ solution $(30 \mathrm{~mL}, 20 \%)$. The solution was left to stand at $0{ }^{\circ} \mathrm{C}$ overnight. The precipitate was collected, washed and dried to give the crude acids.

2-(7-Aminoquinolin-8-ylthio)acetic acid (8a). White plates; $82 \%, \mathrm{mp} 110-12{ }^{\circ} \mathrm{C}$ (from benzene); IR ( $\mathrm{KBr}$ ) $v_{\max }$ $3420,3348,3070,2930,2760,1720,1600,1580,1460,1438,1385,1220,748 \mathrm{~cm}^{-1} ;{ }^{1} \mathrm{H} \mathrm{NMR}\left(400 \mathrm{MHz} \mathrm{CDCl}_{3}\right.$, ppm), $\delta 3.95\left(2 \mathrm{H}, \mathrm{s}, \mathrm{CH}_{2}\right), 4.35\left(2 \mathrm{H}, \mathrm{s}, \mathrm{NH}_{2}\right), 7.36(1 \mathrm{H}, \mathrm{q}, J 4.4,3.6$ and $4.8 \mathrm{~Hz}), 7.54(1 \mathrm{H}, \mathrm{d}, J 8.0 \mathrm{~Hz}), 8.00(1 \mathrm{H}, \mathrm{td}$, J 1.6, 1.2, 4.4, 2.0 and $1.2 \mathrm{~Hz}), 8.50(1 \mathrm{H}, \mathrm{dd}, J 1.2$ and $1.6 \mathrm{~Hz}), 8.87(1 \mathrm{H}, \mathrm{dd}, J 1.6$ and $1.6 \mathrm{~Hz}), 10.39(1 \mathrm{H}, \mathrm{s}, \mathrm{COOH})$; ${ }^{13} \mathrm{C} \mathrm{NMR}\left(100 \mathrm{MHz} \mathrm{CDCl}_{3}, \delta, \mathrm{ppm}\right): 32.5,122.1,126.6,127.4,127.9,130.8,134.4(2 \mathrm{C}), 144.8,148.7$, 171.3. Anal. Calcd. for $\mathrm{C}_{11} \mathrm{H}_{10} \mathrm{~N}_{2} \mathrm{O}_{2} \mathrm{~S}$ (234); C, 65.41; H, 4.27; N, 11.96; S, 13.67. Found; C, 65.45; H, 4.39; N, 11.84; S, $13.78 \%$. 3-(7-Aminoquinolin-8-ylthio)propanoic acid (8b). White plates; 76\%, mp 174-6 ${ }^{\circ} \mathrm{C}$ (from AcOEt); IR (KBr) $v_{\max }$ $3380,3320,3050,2960,2784,1718,1610,1590,1470,1445,1381,1230,760 \mathrm{~cm}^{-1} .{ }^{1} \mathrm{H} \mathrm{NMR}\left(400 \mathrm{MHz} \mathrm{CDCl}_{3}\right.$, ppm), $\delta 2.75\left(1 \mathrm{H}, \mathrm{t}, J 6.4 \mathrm{~Hz}, \mathrm{CH}_{2}\right), 3.35\left(1 \mathrm{H}, \mathrm{t}, J 6.4 \mathrm{~Hz}, \mathrm{CH}_{2}\right), 4.51\left(2 \mathrm{H}, \mathrm{s}, \mathrm{NH}_{2}\right), 7.42(1 \mathrm{H}, \mathrm{q}, J 4.8,3.2$ and $4.8 \mathrm{~Hz})$, $7.54\left(1 \mathrm{H}, \mathrm{d}, J 8.0 \mathrm{~Hz}, \mathrm{CH}_{2}\right), 7.98(1 \mathrm{H}, \mathrm{app} \mathrm{q}, J 1.6,2.0$ and $6.4 \mathrm{~Hz}), 8.49(1 \mathrm{H}, \mathrm{dd}, J 1.6$ and $1.6 \mathrm{~Hz}), 8.87(1 \mathrm{H}, \mathrm{dd}, J$ 1.6 and $1.6 \mathrm{~Hz}), 10.80(1 \mathrm{H}, \mathrm{s}, \mathrm{COOH}) ;{ }^{13} \mathrm{C} \mathrm{NMR}\left(100 \mathrm{MHz}, \mathrm{CDCl}_{3}, \delta, \mathrm{ppm}\right): 28.6,34.2,122.1,126.6,127.4,127.9$, 130.8, 134.4 (2C), 144.8, 148.7, 175.2. Anal. Calcd. for $\mathrm{C}_{12} \mathrm{H}_{12} \mathrm{~N}_{2} \mathrm{O}_{2} \mathrm{~S}$ (248); C, 58.06; H, 4.83; N, 11.29; S, 12.90. Found; C, 58.14; H, 4.70; N, 11.41; S, $12.86 \%$.

4-(7-Aminoquinolin-8-ylthio)butanoic acid (8c). Pale yellow cryatals; 88\%; mp 174-6 ${ }^{\circ} \mathrm{C}$ (from AcOEt); IR (KBr) $V_{\max } 3410,3350,3040,2954,2757,1720,1605,1590,1465,1440,1390,1230,742 \mathrm{~cm}^{-1} .{ }^{1} \mathrm{H} \mathrm{NMR}(400 \mathrm{MHz}$, $\left.\mathrm{CDCl}_{3}, \mathrm{ppm}\right), \delta 1.86\left(2 \mathrm{H}, \mathrm{sp}, J 7.2,7.2,7.6\right.$ and $\left.7.6 \mathrm{~Hz}, \mathrm{CH}_{2}\right), 2.39\left(1 \mathrm{H}, \mathrm{t}, J 7.2\right.$ and $\left.7.6 \mathrm{~Hz}, \mathrm{CH}_{2}\right), 3.14(1 \mathrm{H}, \mathrm{t}, J 7.6$ and $\left.7.6 \mathrm{~Hz}, \mathrm{CH}_{2}\right), 4.62\left(2 \mathrm{H}, \mathrm{s}, \mathrm{NH}_{2}\right), 7.42(1 \mathrm{H}, \mathrm{q}, J 4.8,3.2$ and $4.8 \mathrm{~Hz}), 7.54(1 \mathrm{H}, \mathrm{d}, J 8.0 \mathrm{~Hz}), 7.98(1 \mathrm{H}, \mathrm{td}, J 2.0$, $1.2,2.8$ and $1.6 \mathrm{~Hz}), 8.50(1 \mathrm{H}$, app t, $J 6.8$ and $1.6 \mathrm{~Hz}), 8.87(1 \mathrm{H}, \mathrm{dd}, J 2.0$ and $1.6 \mathrm{~Hz}), 10.73(1 \mathrm{H}, \mathrm{s}, \mathrm{COOH}) ;{ }^{13} \mathrm{C}$ NMR (100 MHz, $\left.\mathrm{CDCl}_{3}, \delta, \mathrm{ppm}\right): 24.8,32.9,33.2,122.1,126.6,127.4,127.9,130.8,134.4(2 \mathrm{C}), 144.8,148.7$, 177.6. Anal. Calcd. for $\mathrm{C}_{13} \mathrm{H}_{14} \mathrm{~N}_{2} \mathrm{O}_{2} \mathrm{~S}$ (262); C, 59.54; H, 5.34; N, 10.68; S, 12.21. Found; C, 59.52; H, 5.48; N, 10.72; S, $12.05 \%$.

General Procedure for the synthesis of 7-bromoquinolin-8-ylthio)alkanoic acids (9a-c). To a stirred solution of amino acid $8 \mathbf{a}$ or $\mathbf{b}$ or $\mathbf{c}(50 \mathrm{mmol})$ in water $(50 \mathrm{~mL})$ was added concentrated $\mathrm{HCl}(12 \mathrm{~mL})$ at room temperature. The flask was warmed on a hot plate until no solids remained. The solution was then cooled in an ice bath and an aqueous solution of $\mathrm{NaNO}_{2}(3.6 \mathrm{~g}, 53 \mathrm{mmol})$ in water $(25 \mathrm{~mL})$ was added slowly with stirring. The resulting solution was stirred for 10 minutes. A solution of $\mathrm{KBr}(8.5 \mathrm{~g}, 55 \mathrm{mmol})$ in water $(12 \mathrm{~mL})$ was added with occasional swirling and the solution was then heated at $100{ }^{\circ} \mathrm{C}$ for $30 \mathrm{~min}$. The reaction mixture was cooled to 
ambient temperature and the precipitate was filtered and washed with water to afford the crude acids $\mathbf{9 a}$ or $\mathbf{b}$ or c. Purifications, yields and spectral data are given in the following:

2-(7-Bromoquinolin-8-ylthio)acetic acid (9a). Yellow solid; 82\%, mp 139-41 ${ }^{\circ} \mathrm{C}$ (from AcOEt); IR (KBr) $v_{\max } 3010$, 2930, 2645, 1715, 1600, 1590, 1470, 1440, 1375, 1248, $757 \mathrm{~cm}^{-1} ;{ }^{1} \mathrm{H}$ NMR (400 MHz, CDCl $\left.3, \mathrm{ppm}\right), \delta 3.95(2 \mathrm{H}, \mathrm{s}$, $\left.\mathrm{CH}_{2}\right), 7.30(1 \mathrm{H}, \mathrm{q}, J 4.8,3.2$ and $4.0 \mathrm{~Hz}), 7.81(1 \mathrm{H}, \mathrm{d}, J 8.4 \mathrm{~Hz}), 8.07(1 \mathrm{H}, \mathrm{td}, J 1.6,1.6,1.2,1.2$ and $2.0 \mathrm{~Hz}), 8.40$ $(1 \mathrm{H}$, app t, J 7.2 and $1.6 \mathrm{~Hz}), 8.89(1 \mathrm{H}, \mathrm{dd}, J 2.4$ and $1.6 \mathrm{~Hz}), 10.52(1 \mathrm{H}, \mathrm{s}, \mathrm{COOH}) ;{ }^{13} \mathrm{C} \mathrm{NMR}(100 \mathrm{MHz}, \mathrm{CDCl}, \delta$, ppm): $32.5,119.7,122.1,126.6,127.9,130.8,131.8,134.4,144.8,148.7,171.3$. Anal. Calcd. for $\mathrm{C}_{11} \mathrm{H}_{8} \mathrm{BrNO}_{2} \mathrm{~S}$ (297); C, 44.44; H, 2.69; Br, 26.59; N, 4.71; S, 10.77. Found; C, 44.59; H, 2.75; Br, 26.46; N, 4.75; S, $10.61 \%$.

3-(7-Bromoquinolin-8-ylthio)propanoic acid (9b). Yellow crystals; 74\%, mp 154-6 ${ }^{\circ} \mathrm{C}$ (from benzene); IR (KBr) $V_{\max } 3035,2960,2585,1720,1590,1464,1440,1390,1235,748 \mathrm{~cm}^{-1} ;{ }^{1} \mathrm{H} \mathrm{NMR}\left(400 \mathrm{MHz}, \mathrm{CDCl}_{3}, \mathrm{ppm}\right), \delta 2.71$ $\left(2 \mathrm{H}, \mathrm{t}, J 6.4\right.$ and $\left.6.4 \mathrm{~Hz}, \mathrm{CH}_{2}\right), 3.32\left(2 \mathrm{H}, \mathrm{t}, J 6.8\right.$ and $\left.6.4 \mathrm{~Hz}, \mathrm{CH}_{2}\right), 7.29(1 \mathrm{H}, \mathrm{q}, J 4.4,3.2$ and $4.4 \mathrm{~Hz}), 7.81(1 \mathrm{H}, \mathrm{d}, J$ $8.4 \mathrm{~Hz}), 8.06(1 \mathrm{H}, \mathrm{td}, J$ 2.0, 1.2, 3.6, 2.0 and $1.2 \mathrm{~Hz}), 8.40(1 \mathrm{H}, \mathrm{dd}, J 1.2$ and $1.6 \mathrm{~Hz}), 8.89(1 \mathrm{H}$, app dd, J $1.6 \mathrm{and}$ $3.2 \mathrm{~Hz}), 9.77(1 \mathrm{H}, \mathrm{s}, \mathrm{COOH}) ;{ }^{13} \mathrm{C} \mathrm{NMR}\left(100 \mathrm{MHz}_{\mathrm{CDCl}}, \delta, \mathrm{ppm}\right): 28.7,34.2,119.7,122.1,126.6,127.9,130.8$, 131.8, 134.4, 144.8, 148.7, 175.2. Anal. Calcd. for $\mathrm{C}_{12} \mathrm{H}_{10} \mathrm{BrNO}_{2} \mathrm{~S}$ (311); C, 46.30; H, 3.21; Br, 25.40; N, 4.50; S, 10.28. Found; C, 46.39; $\mathrm{H}, 3.34 ; \mathrm{Br}, 25.24 ; \mathrm{N}, 4.61 ; \mathrm{S}, 10.30 \%$.

4-(7-Bromoquinolin-8-ylthio)butanoic acid (9c). white plates; 78\%, mp $125-7{ }^{\circ} \mathrm{C}$ (from benzene); IR ( $\mathrm{KBr}$ ) $v_{\max }$ $3044,2960,2570,1720,1590,1560,1480,1440,1380,1250,740 \mathrm{~cm}^{-1} ;{ }^{1} \mathrm{H} \mathrm{NMR}\left(400 \mathrm{MHz}, \mathrm{CDCl}_{3}, \mathrm{ppm}\right), \delta 1.86$ $\left(2 \mathrm{H}, \mathrm{sp}, J 3.2,6.4,7.6\right.$ and $\left.7.6 \mathrm{~Hz}, \mathrm{CH}_{2}\right), 2.40\left(2 \mathrm{H}, \mathrm{t}, J 7.2\right.$ and $\left.7.6 \mathrm{~Hz}, \mathrm{CH}_{2}\right), 3.13\left(2 \mathrm{H}, \mathrm{t}, J 7.6\right.$ and $\left.7.6 \mathrm{~Hz}, \mathrm{CH}_{2}\right), 7.29$ $(1 \mathrm{H}, \mathrm{q}, J 4.8,3.2$ and $4.8 \mathrm{~Hz}), 7.81(1 \mathrm{H}, \mathrm{d}, J 8.4 \mathrm{~Hz}), 8.06(1 \mathrm{H}, \mathrm{td}, J 2.0,1.2,3.6,2.0$ and $1.2 \mathrm{~Hz}), 8.40(1 \mathrm{H}$, app t, J 6.8 and $1.6 \mathrm{~Hz}), 8.89(1 \mathrm{H}$, app dd, $J 1.6$ and $3.2 \mathrm{~Hz}), 10.20(1 \mathrm{H}, \mathrm{s}, \mathrm{COOH}) ;{ }^{13} \mathrm{C} \mathrm{NMR}(100 \mathrm{MHz}, \mathrm{CDCl}, \delta, \mathrm{ppm})$ : 24.8, 32.9, 33.2, 119.7, 122.1, 126.6, 127.9, 130.8, 131.8, 134.4, 144.8, 148.7, 177.6. Anal. Calcd. for $\mathrm{C}_{13} \mathrm{H}_{12} \mathrm{BrNO}_{2} \mathrm{~S}$ (325); C, 48.00; H, 3.69; $\mathrm{Br}, 24.30 ; \mathrm{N}, 4.30 ; \mathrm{S}, 9.84$. Found; C, 48.13; H, 3.52; Br, 24.28; N, 4.34; S, $9.78 \%$.

General procedure for arylation using (7-bromoquinolin-8-ylthio)carboxylic acids (9a-c). A mixture of bromocarboxylic acids $9 \mathbf{a}$ or $\mathbf{b}$ or $\mathbf{c}(20 \mathrm{mmol}), \mathrm{K}_{2} \mathrm{CO}_{3}(4.1 \mathrm{~g}, 50 \mathrm{mmol})$, tosylated arylamine (PhNHTs or $\mathrm{N}$-tosylpyridin2-amine or Ts $\left.\mathrm{NHCH}_{2} \mathrm{Ph}\right)(22 \mathrm{mmol})$ in DMSO $(20 \mathrm{~mL})$ was heated with efficient stirring for $10 \mathrm{~h}$ at $120-30{ }^{\circ} \mathrm{C}$. after which TLC analysis (EtOAc) indicated that the reaction was complete, the solution was cooled and treated with $\mathrm{NaOH}$ solution $(40 \mathrm{~mL}, 10 \%)$. The solution was refluxed for $10 \mathrm{~min}$ then filtered by suction. The filtrate was concentrated and acidified with aqueous $\mathrm{HCl}$ solution $(40 \mathrm{~mL}, 10 \%)$. The resulted precipitate was filtered, washed with water, dried to give the crude acids 10a-i. The yields and spectral data are given in the following: 2-(7-(N-Phenyl-N-tosylamino)quinolin-8-ylthio)acetic acid (10a). White crystals; 79\%, mp $182-4{ }^{\circ} \mathrm{C}$ (from benzene); IR (KBr) $v_{\max } 3085,2975,2585,1720,1585,1455,1440,1390,1237,748 \mathrm{~cm}^{-1} ;{ }^{1} \mathrm{H} \mathrm{NMR}(400 \mathrm{MHz}$, $\left.\mathrm{CDCl}_{3}, \mathrm{ppm}\right), \delta 2.33\left(3 \mathrm{H}, \mathrm{s}, \mathrm{CH}_{3}\right), 3.97\left(2 \mathrm{H}, \mathrm{s}, \mathrm{CH}_{2}\right), 7.06(1 \mathrm{H}, \mathrm{d}, J 8.4 \mathrm{~Hz}), 7.12(2 \mathrm{H}, \mathrm{dd}, J 1.2$ and $1.2 \mathrm{~Hz}), 7.19(1 \mathrm{H}$, app quin, J 6.4, 1.2, 1.6 and $6.0 \mathrm{~Hz}), 7.31(1 \mathrm{H}, \mathrm{d}, J 2.4 \mathrm{~Hz}), 7.33(2 \mathrm{H}, \mathrm{t}, J 2.8$ and $4.8 \mathrm{~Hz}), 7.49(2 \mathrm{H}, \mathrm{d}, J 8.0 \mathrm{~Hz}), 7.65$ $(2 \mathrm{H}, \mathrm{t}, J \mathrm{~J} .6,0.8$ and $7.6 \mathrm{~Hz}), 8.06(1 \mathrm{H}, \mathrm{dd}, J 1.6$ and $1.2 \mathrm{~Hz}), 8.21(1 \mathrm{H}$, app dt, J 1.6, 2.0, 2.0 and $1.2 \mathrm{~Hz}), 8.86(1 \mathrm{H}$, dd, $J 2.0$ and $1.2 \mathrm{~Hz}), 10.46(1 \mathrm{H}, \mathrm{s}, \mathrm{COOH}) ;{ }^{13} \mathrm{C} \mathrm{NMR}\left(100 \mathrm{MHz}, \mathrm{CDCl}_{3}, \delta, \mathrm{ppm}\right): 21.2,32.5,117.2,122.1,122.9$ (2C), 124.7, $125.3(2 \mathrm{C}), 126.6,127.4(2 \mathrm{C}), 127.9,129.7$ (2C), 130.8, 134.4, 135.0, 144.2, 144.8, 148.7, 171.3. Anal. Calcd. for $\mathrm{C}_{24} \mathrm{H}_{20} \mathrm{~N}_{2} \mathrm{O}_{4} \mathrm{~S}_{2}$ (464); C, 62.06; $\mathrm{H}, 4.31 ; \mathrm{N}, 6.03 ; \mathrm{S}, 13.79$. Found; $\mathrm{C}, 62.02 ; \mathrm{H}, 4.38 ; \mathrm{N}, 6.11 ; \mathrm{S}, 13.65 \%$.

2-(7-(N-(Pyridin-2-yl)-N-tosylamino)quinolin-8-ylthio)acetic acid (10b). White crystals; $84 \%, \mathrm{mp} 122-4{ }^{\circ} \mathrm{C}$ (from benzene); IR (KBr) $v_{\max } 3074,2983,2580,1718,1593,1563,1475,1440,1380,1244,745 \mathrm{~cm}^{-1} ;{ }^{1} \mathrm{H} N M R(400$ $\left.\mathrm{MHz}, \mathrm{CDCl}_{3}, \mathrm{ppm}\right), \delta 2.33\left(3 \mathrm{H}, \mathrm{s}, \mathrm{CH}_{3}\right), 3.96\left(2 \mathrm{H}, \mathrm{s}, \mathrm{CH}_{2}\right), 7.05(1 \mathrm{H}, \mathrm{d}, J 8.4 \mathrm{~Hz}), 7.21(1 \mathrm{H}, \mathrm{app} \mathrm{dt}, J 1.2,0.8,1.2$, and 3.6 Hz), $7.31(2 \mathrm{H}, \mathrm{d}, J 8.4 \mathrm{~Hz}), 7.38(1 \mathrm{H}, \mathrm{q}, J 4.8,3.2$ and $4.8 \mathrm{~Hz}), 7.49(1 \mathrm{H}, \mathrm{d}, J 8.0 \mathrm{~Hz}), 7.87-7.90(2 \mathrm{H}, \mathrm{m}), 8.07$ $(1 \mathrm{H}, \mathrm{dd}, J 1.6,7.2$ and $2.0 \mathrm{~Hz}), 8.21(1 \mathrm{H}$, app dt, J 2.0, 1.2, 4.8 and $1.6 \mathrm{~Hz}), 8.44(1 \mathrm{H}, \mathrm{dd}, J 2.0$ and $1.2 \mathrm{~Hz}), 8.86$ $(1 \mathrm{H}, \mathrm{dd}, J 2.0$ and $1.6 \mathrm{~Hz}), 11.16(1 \mathrm{H}, \mathrm{s}, \mathrm{COOH}) ;{ }^{13} \mathrm{C} \mathrm{NMR}\left(100 \mathrm{MHz}, \mathrm{CDCl}_{3}, \delta, \mathrm{ppm}\right): 21.2,32.5,111.4,117.2$, 
118.8, 122.1, 126.6, 127.4 (2C), 127.9, 129.7 (2C), 130.8, 134.4, 135.0, 138.1, 140.4, 144.2, 144.8, 148.3, 148.7, 153.9, 171.3. Anal. Calcd. for $\mathrm{C}_{23} \mathrm{H}_{19} \mathrm{~N}_{3} \mathrm{O}_{4} \mathrm{~S}_{2}$ (465); C, 59.35; $\mathrm{H}, 4.08 ; \mathrm{N}, 9.03 ; \mathrm{S}, 13.76$. Found; $\mathrm{C}, 59.33 ; \mathrm{H}, 4.14 ; \mathrm{N}$, 9.10; S, 13.55\%.

2-(7-(N-Benzyl-N-tosylamino)quinolin-8-ylthio)acetic acid (10c). Yellow needles; 82\%, mp $140-2{ }^{\circ} \mathrm{C}$ (from acetone); IR (KBr) $v_{\max } 3030,2980,2750,1720,1605,1581,1440,1375,1237,750 \mathrm{~cm}^{-1} ;{ }^{1} \mathrm{H} \mathrm{NMR}(400 \mathrm{MHz}$, $\left.\mathrm{CDCl}_{3}, \mathrm{ppm}\right), \delta 2.32\left(3 \mathrm{H}, \mathrm{s}, \mathrm{CH}_{3}\right), 3.95\left(2 \mathrm{H}, \mathrm{s}, \mathrm{CH}_{2}\right), 5.15\left(2 \mathrm{H}, \mathrm{s}, \mathrm{CH}_{2}\right), 7.01-7.06(3 \mathrm{H}, \mathrm{m}), 7.25-7.42(6 \mathrm{H}, \mathrm{m}), 7.40$ $(2 \mathrm{H}, \mathrm{d}, J 8.0 \mathrm{~Hz}), 7.80(1 \mathrm{H}$, two t, J 2.0, 1.6 and $1.6 \mathrm{~Hz}), 8.05(1 \mathrm{H}, \mathrm{dd}, J 1.6$ and $2.0 \mathrm{~Hz}), 8.83(1 \mathrm{H}, \mathrm{dd}, J 1.6 \mathrm{and} 1.6$ $\mathrm{Hz}), 10.82(1 \mathrm{H}, \mathrm{s}, \mathrm{COOH}) ;{ }^{13} \mathrm{C} \mathrm{NMR}\left(100 \mathrm{MHz} \mathrm{CDCl}_{3}, \delta, \mathrm{ppm}\right): 21.2,32.5,55.8,117.2,122.1,126.6,127.4(2 \mathrm{C})$, 127.7 (2C), 127.9, 128.5 (2C), 128.9, 129.7 (2C), 134.4, 134.8, 137.3, 140.4, 144.2, 144.8, 148.7, 171.3. Anal. Calcd. for $\mathrm{C}_{25} \mathrm{H}_{22} \mathrm{~N}_{2} \mathrm{O}_{4} \mathrm{~S}_{2}$ (478); C, 62.76; $\mathrm{H}, 4.60 ; \mathrm{N}, 5.85 ; \mathrm{S}, 13.38$. Found; $\mathrm{C}, 62.66 ; \mathrm{H}, 4.64 ; \mathrm{N}, 5.79 ; \mathrm{S}, 13.42 \%$. 3-(7-(N-Phenyl-N-tosylamino)quinolin-8-ylthio)propanoic acid (10d). White crystals; $78 \%, \mathrm{mp} 95-7^{\circ} \mathrm{C}$ (from acetone); IR (KBr) $v_{\max } 3040,2960,2620,1720,1610,1585,1460,1445,1385,1270,750 \mathrm{~cm}^{-1} ;{ }^{1} \mathrm{H}$ NMR (400 $\left.\mathrm{MHz}_{2} \mathrm{CDCl}_{3}, \mathrm{ppm}\right), \delta 2.33\left(3 \mathrm{H}, \mathrm{s}, \mathrm{CH}_{3}\right), 2.74\left(2 \mathrm{H}, \mathrm{t}, J 6.8 \mathrm{~Hz}, \mathrm{CH}_{2}\right), 3.41\left(2 \mathrm{H}, \mathrm{t}, J 6.8\right.$ and $\left.6.4 \mathrm{~Hz}, \mathrm{CH}_{2}\right), 7.04(1 \mathrm{H}, \mathrm{d}, J$ $8.4 \mathrm{~Hz}), 7.12(2 \mathrm{H}, \mathrm{dd}, J 1.2$ and $1.6 \mathrm{~Hz}), 7.19-7.23(1 \mathrm{H}, \mathrm{m}), 7.31(3 \mathrm{H}$, app t, J 6.4, 1.2 and $6.4 \mathrm{~Hz}), 7.49(2 \mathrm{H}, \mathrm{d}, J 8.0$ $\mathrm{Hz}), 7.65(2 \mathrm{H}, \mathrm{t}, J \mathrm{~J} .6,0.8$ and $7.6 \mathrm{~Hz}), 8.06(1 \mathrm{H}, \mathrm{dd}, J 1.6$ and $1.6 \mathrm{~Hz}), 8.21(1 \mathrm{H}$, two t, J 2.0, 1.2, 4.8, 1.6 and 2.0 $\mathrm{Hz}), 8.86(1 \mathrm{H}, \mathrm{dd}, J 2.0$ and $1.6 \mathrm{~Hz}), 10.53(1 \mathrm{H}, \mathrm{s}, \mathrm{COOH}) ;{ }^{13} \mathrm{C} \mathrm{NMR}\left(100 \mathrm{MHz}, \mathrm{CDCl}_{3}, \delta, \mathrm{ppm}\right): 21.2,28.6,34.29$, $117.2,122.1,122.9(2 \mathrm{C}), 124.7,125.3(2 \mathrm{C}), 126.6,127.4(2 \mathrm{C}), 127.9,129.7(2 \mathrm{C}), 130.8,134.4,135.0,144.2,144.8$, 148.7, 175.2. Anal. Calcd. for $\mathrm{C}_{25} \mathrm{H}_{22} \mathrm{~N}_{2} \mathrm{O}_{4} \mathrm{~S}_{2}$ (478); C, 62.76; $\mathrm{H}, 4.60 ; \mathrm{N}, 5.85 ; \mathrm{S}, 13.38$. Found; C, 62.74; $\mathrm{H}, 4.69 ; \mathrm{N}$, $5.93 ;$ S, $13.47 \%$.

3-(7-(N-(Pyridin-2-yl)-N-tosylamino)quinolin-8-ylthio)propanoic acid (10e). White plates; $85 \%, \mathrm{mp} 113-5{ }^{\circ} \mathrm{C}$ (from benzene); IR (KBr) $v_{\max } 3025,2980,2560,1720,1585,1560,1495,1438,1374,1240,748 \mathrm{~cm}^{-1} ;{ }^{1} \mathrm{H} \mathrm{NMR}$ $\left(400 \mathrm{MHz}_{\mathrm{CDCl}}, \mathrm{ppm}\right), \delta 2.33\left(3 \mathrm{H}, \mathrm{s}, \mathrm{CH}_{3}\right), 2.74\left(2 \mathrm{H}, \mathrm{t}, \mathrm{J} 6.8\right.$ and $\left.6.4 \mathrm{~Hz}, \mathrm{CH}_{2}\right), 3.41\left(2 \mathrm{H}, \mathrm{t}, J 6.8\right.$ and $\left.6.4 \mathrm{~Hz}, \mathrm{CH}_{2}\right)$, $7.04(1 \mathrm{H}, \mathrm{d}, J 8.8 \mathrm{~Hz}), 7.20(1 \mathrm{H}, \mathrm{ddd}, J 3.6,1.2,0.8,1.2,3.6$ and $2.0 \mathrm{~Hz}), 7.31(2 \mathrm{H}, \mathrm{d}, J 7.6 \mathrm{~Hz}), 7.39(1 \mathrm{H}, \mathrm{q}, J 3.6$, 3.2 and $3.6 \mathrm{~Hz}), 7.49(2 \mathrm{H}, \mathrm{d}, J 8.0 \mathrm{~Hz}), 7.85-7.91(2 \mathrm{H}, \mathrm{m}), 8.06(1 \mathrm{H}, \mathrm{dd}, J 1.6$ and $1.2 \mathrm{~Hz}), 8.21(1 \mathrm{H}, \mathrm{two} \mathrm{t}, J 2.0$, 1.2, 2.0 and $1.6 \mathrm{~Hz}), 8.44(1 \mathrm{H}, \mathrm{dd}, J 1.6$ and $1.2 \mathrm{~Hz}), 8.86(1 \mathrm{H}, \mathrm{dd}, J 2.0$ and $2.0 \mathrm{~Hz}), 10.41(1 \mathrm{H}, \mathrm{s}, \mathrm{COOH}) ;{ }^{13} \mathrm{C} \mathrm{NMR}$ $\left(100 \mathrm{MHz}_{\mathrm{CDCl}}, \delta, \mathrm{ppm}\right): 21.2,28.6,34.2,111.4,117.2,118.8,122.1,126.6,127.4(2 \mathrm{C}), 127.9,129.7$ (2C), 130.8 , $134.4,135.0,138.1,140.4,144.2,144.8,148.3,148.7,153.9,175.2$. Anal. Calcd. for $\mathrm{C}_{24} \mathrm{H}_{21} \mathrm{~N}_{3} \mathrm{O}_{4} \mathrm{~S}_{2}$ (479); C, 62.12; $\mathrm{H}, 4.38 ; \mathrm{N}, 8.76 ; \mathrm{S}, 13.36$. Found; C, 62.31; H, 4.48; N, 8.85; S, $13.32 \%$.

3-(7-(N-Benzyl-N-tosylamino)quinolin-8-ylthio)propanoic acid (10f). White needles; $81 \%, \mathrm{mp} 110-12^{\circ} \mathrm{C}$ (from ethanol); IR (KBr) $v_{\max } 3050,2910,2670,1722,1590,1580,1477,1445,1395,1240,755 \mathrm{~cm}^{-1} ;{ }^{1} \mathrm{H} N M R(400$ $\left.\mathrm{MHz}_{\mathrm{CDCl}}, \mathrm{ppm}\right), \delta 2.32\left(3 \mathrm{H}, \mathrm{s}, \mathrm{CH}_{3}\right), 2.76\left(2 \mathrm{H}, \mathrm{t}, J 6.8\right.$ and $\left.3.2 \mathrm{~Hz}, \mathrm{CH}_{2}\right), 3.40\left(2 \mathrm{H}, \mathrm{t}, J 6.8 \mathrm{~Hz}, \mathrm{CH}_{2}\right), 5.13(2 \mathrm{H}, \mathrm{s}$, $\left.\mathrm{CH}_{2}\right), 7.00-7.06(\mathrm{H}, \mathrm{m}), 7.24-7.33(6 \mathrm{H}, \mathrm{m}), 7.40(2 \mathrm{H}, \mathrm{d}, J 8.0 \mathrm{~Hz}), 7.80(1 \mathrm{H}$, two t, J 3.2, 4.2, 1.6 and $1.6 \mathrm{~Hz}), 8.04$ $(1 \mathrm{H}, \mathrm{dd}, J 1.6$ and $2.0 \mathrm{~Hz}), 8.83(1 \mathrm{H}, \mathrm{dd}, J 1.6$ and $1.6 \mathrm{~Hz}), 10.70(1 \mathrm{H}, \mathrm{s}, \mathrm{COOH}) ;{ }^{13} \mathrm{C} \mathrm{NMR}\left(100 \mathrm{MHz}, \mathrm{CDCl}_{3}, \delta, \mathrm{ppm}\right)$ : 21.2, 28.6, 34.2, 55.8, 117.2, 122.1, 126.6, 127.4 (2C), 127.7 (2C), 127.9, 128.5 (2C), 128.9, 129.7 (2C), 134.4, 134.8, 137.3, 144.2, 144.8, 148.7, 175.2. Anal. Calcd. for $\mathrm{C}_{26} \mathrm{H}_{24} \mathrm{~N}_{2} \mathrm{O}_{4} \mathrm{~S}_{2}(492) ; \mathrm{C}, 63.41 ; \mathrm{H}, 4.87 ; \mathrm{N}, 5.69 ; \mathrm{S}, 13.00$. Found; C, 63.49; $\mathrm{H}, 4.82 ; \mathrm{N}, 5.74 ; \mathrm{S}, 12.88 \%$.

4-(7-(N-Phenyl-N-tosylamino)quinolin-8-ylthio)butanoic acid (10g). White cryatals; $80 \%, \mathrm{mp} 158-60{ }^{\circ} \mathrm{C}$ (from AcOEt); IR (KBr) $v_{\max } 3045,2965,2620,1720,1587,1550,1440,1435,1385,1290,740 \mathrm{~cm}^{-1} ;{ }^{1} \mathrm{H} \mathrm{NMR}(400 \mathrm{MHz}$, $\left.\mathrm{CDCl}_{3}, \mathrm{ppm}\right), \delta 1.87\left(2 \mathrm{H}\right.$, quin, J 7.6, 7.6, 7.2 and $\left.7.6 \mathrm{~Hz}, \mathrm{CH}_{2}\right), 2.33\left(3 \mathrm{H}, \mathrm{s}, \mathrm{CH}_{3}\right), 2.39\left(2 \mathrm{H}, \mathrm{t}, J 7.6\right.$ and $\left.7.2 \mathrm{~Hz}, \mathrm{CH}_{2}\right)$, $3.20\left(2 \mathrm{H}, \mathrm{t}, J 7.6 \mathrm{~Hz}, \mathrm{CH}_{2}\right), 7.04(1 \mathrm{H}, \mathrm{d}, J 8.8 \mathrm{~Hz}), 7.12(2 \mathrm{H}, \mathrm{dd}, J$ 1.2, 2,8 and $1.6 \mathrm{~Hz}), 7.21-7.23(1 \mathrm{H}, \mathrm{m}), 7.31-7.35$ $(3 \mathrm{H}, \mathrm{m}), 7.49(2 \mathrm{H}, \mathrm{d}, J 8.0 \mathrm{~Hz}), 7.65(2 \mathrm{H}, \mathrm{t}, J 7.6$ and $8.4 \mathrm{~Hz}), 8.06(1 \mathrm{H}, \mathrm{dd}, J 1.6 \mathrm{and} 1.6 \mathrm{~Hz}), 8.20(1 \mathrm{H}, \mathrm{two} \mathrm{t}, J 1.6$, 1.6, 1.6 and $1.6 \mathrm{~Hz}), 8.86(1 \mathrm{H}, \mathrm{dd}, J 2.0$ and $1.6 \mathrm{~Hz}), 11.06(1 \mathrm{H}, \mathrm{s}, \mathrm{COOH}) ;{ }^{13} \mathrm{C} \mathrm{NMR}(100 \mathrm{MHz}, \mathrm{CDCl}, \delta, \mathrm{ppm})$ : 21.2, 24.8, 32.9, 33.2, 117.2, 122.1, 122.9 (2C), 124.7, 125.3 (2C), 126.6, $127.4(2 \mathrm{C}), 127.9,129.7(2 \mathrm{C}), 130.8$, 
134.4, 135.0, 144.2, 144.8, 148.7, 177.6. Anal. Calcd. for $\mathrm{C}_{26} \mathrm{H}_{24} \mathrm{~N}_{2} \mathrm{O}_{4} \mathrm{~S}_{2}(492) ; \mathrm{C}, 63.41 ; \mathrm{H}, 4.87 ; \mathrm{N}, 5.69 ; \mathrm{S}, 13.00$. Found; C, 63.48; H, 4.95; N, 5.62; S, $13.13 \%$.

4-(7-(N-(Pyridin-2-yl)-N-tosylamino)quinolin-8-ylthio)butanoic acid (10h). White crystals; $82 \%, \mathrm{mp} 140-2{ }^{\circ} \mathrm{C}$ (from benzene); IR (KBr) $v_{\max } 3045,2985,2790,1720,1605,1585,1440,1370,1284,754 \mathrm{~cm}^{-1} ;{ }^{1} \mathrm{H}$ NMR (400 $\left.\mathrm{MHz}_{2} \mathrm{CDCl}_{3}, \mathrm{ppm}\right), \delta 1.87\left(2 \mathrm{H}\right.$, quin, J 7.2, 7.2, 7.6 and 7.6 Hz, $\left.\mathrm{CH}_{2}\right), 2.33\left(3 \mathrm{H}, \mathrm{s}, \mathrm{CH}_{3}\right), 2.39(2 \mathrm{H}, \mathrm{t}, J 7.6 \mathrm{and} 7.2 \mathrm{~Hz}$, $\left.\mathrm{CH}_{2}\right), 3.20\left(2 \mathrm{H}, \mathrm{t}, J 7.6\right.$ and $\left.7.6 \mathrm{~Hz}, \mathrm{CH}_{2}\right), 7.04(1 \mathrm{H}, \mathrm{d}, J 8.8 \mathrm{~Hz}), 7.19(1 \mathrm{H}$, ddd, J 2.0, 3.6, 1.2, 0.8, 1.2, 3.6 and 2.0 $\mathrm{Hz}), 7.31(2 \mathrm{H}, \mathrm{d}, J 8.4 \mathrm{~Hz}), 7.39(2 \mathrm{H}, \mathrm{q}, J 4.8,3.2$ and $4.8 \mathrm{~Hz}), 7.49(2 \mathrm{H}, \mathrm{d}, J 8.0 \mathrm{~Hz}), 7.85-7.91(2 \mathrm{H}, \mathrm{m}), 8.06(1 \mathrm{H}$, dd, J 1.6 and $1.2 \mathrm{~Hz}), 8.21(1 \mathrm{H}$, two t, J 2.0, 1.2, 1.2, 2.0 and $1.2 \mathrm{~Hz}), 8.44(1 \mathrm{H}, \mathrm{dd}, J 1.6 \mathrm{and} 1.2 \mathrm{~Hz}), 8.86(1 \mathrm{H}, \mathrm{dd}$, J 2.0 and $1.6 \mathrm{~Hz}$ ), 10.54 (1H, s, $\left.\mathrm{COOH}) ;{ }^{13} \mathrm{C} \mathrm{NMR} \mathrm{(100} \mathrm{MHz,} \mathrm{CDCl}_{3}, \delta, \mathrm{ppm}\right): 21.2,24.8,32.9,33.2,111.4,117.2$, 118.8, 122.1, 126.6, 127.4 (2C), 127.9, 129.7 (2C), 130.8, 134.4, 135.0, 138.1, 140.4, 144.2, 144.8, 148.3, 148.7, 153.9, 177.6. Anal. Calcd. for $\mathrm{C}_{25} \mathrm{H}_{23} \mathrm{~N}_{3} \mathrm{O}_{4} \mathrm{~S}_{2}$ (493); C, 60.85; H, 4.66; N, 8.51; S, 12.98. Found; C, 60.78; $\mathrm{H}, 4.56 ; \mathrm{N}$, $8.58 ; \mathrm{S}, 12.84 \%$.

4-(7-(N-Benzyl-N-tosylamino)quinolin-8-ylthio)butanoic acid (10i). White crystals; 80\%, mp $167-9{ }^{\circ} \mathrm{C}$ (from acetone); IR (KBr) $v_{\max } 3025,2947,2670,1720,1600,1565,1444,1373,1286,758 \mathrm{~cm}^{-1} ;{ }^{1} \mathrm{H} \mathrm{NMR}(400 \mathrm{MHz}$, $\left.\mathrm{CDCl}_{3}, \mathrm{ppm}\right), \delta 1.87\left(2 \mathrm{H}\right.$, quin, J 7.6, 7.2, 7.6 and $\left.7.6 \mathrm{~Hz}, \mathrm{CH}_{2}\right), 2.32\left(3 \mathrm{H}, \mathrm{s}, \mathrm{CH}_{3}\right), 2.39\left(2 \mathrm{H}, \mathrm{t}, J 7.6\right.$ and $\left.7.6 \mathrm{~Hz}, \mathrm{CH}_{2}\right)$, $3.26\left(2 \mathrm{H}, \mathrm{t}, J 7.6\right.$ and $\left.7.6 \mathrm{~Hz}, \mathrm{CH}_{2}\right), 5.13\left(2 \mathrm{H}, \mathrm{s}, \mathrm{CH}_{2}\right), 7.00-7.06(3 \mathrm{H}, \mathrm{m}), 7.24-7.33(6 \mathrm{H}, \mathrm{m}), 7.40(2 \mathrm{H}, \mathrm{d}, J 8.0 \mathrm{~Hz})$, $7.80(1 \mathrm{H}$, two t, J 1.6, 1.6, 1.2, 1.6 and $1.6 \mathrm{~Hz}), 8.04(1 \mathrm{H}$, dd, J 1.6 and $1.6 \mathrm{~Hz}), 8.83(1 \mathrm{H}, \mathrm{dd}, J 1.6 \mathrm{and} 1.6 \mathrm{~Hz})$, $10.85(1 \mathrm{H}, \mathrm{s}, \mathrm{COOH}) ;{ }^{13} \mathrm{C} \mathrm{NMR}\left(100 \mathrm{MHz}, \mathrm{CDCl}_{3}, \delta, \mathrm{ppm}\right): 1.2,24.8,32.9,33.2,55.8,117.2,122.1,126.6,127.4$ (2C), 127.7 (2C), 127.9, 128.5 (2C), 128.9, 129.7 (2C), 134.4, 134.8, 140.4, 144.2, 144.8, 148.7, 177.6. Anal. Calcd. for $\mathrm{C}_{27} \mathrm{H}_{26} \mathrm{~N}_{2} \mathrm{O}_{4} \mathrm{~S}_{2}$ (506); C, 64.03; H, 5.13; N, 5.53; S, 12.64. Found; C, 64.14; H, 5.02; N, 5.48; S, $12.75 \%$.

\section{Friedel-Crafts cycliacylation procedures}

Procedure A. Cycliacylations using $\mathrm{AlCl} / \mathrm{CH}_{3} \mathrm{NO}_{2}$ catalyst. To a solution of $\mathrm{AlCl}_{3}(2.4 \mathrm{mmol})$ in $\mathrm{CH}_{3} \mathrm{NO}_{2}$ (24 mmol) was added a solution of required acid precursor $3 a-i(2.0 \mathrm{mmol})$ in DCM $(10 \mathrm{~mL})$ dropwise with efficient stirring over 10-15 min. The reaction mixture was further stirred for a certain time at room temperature (Tables 1\&2) and decomposed by careful addition of ice-cold $\mathrm{HCl}$ solution $(20 \mathrm{~mL}, 10 \%)$. The residue was extracted with ether $(3 \times 20 \mathrm{~mL})$ and the combined organic phases were washed with $\mathrm{Na}_{2} \mathrm{CO}_{3}(20 \mathrm{~mL}, 10 \%)$, water and dried over anhydrous $\mathrm{MgSO}_{4}$. The solvent was evaporated under reduced pressure to afford the crude products $10 \mathrm{a}-\mathbf{i}$.

Procedure B. Cycliacylations using $\mathrm{P}_{2} \mathrm{O}_{5}$ catalyst. A solution of acid $\mathbf{3 a}-\mathbf{i}(0.5 \mathrm{~g})$ and $\mathrm{P}_{2} \mathrm{O}_{5}(5 \mathrm{~g})$ in dry benzene $(10 \mathrm{~mL})$ was refluxed for the required time (Tables 1\&2) and, after cooling to room temperature, the reaction mixture was diluted with ether $(40 \mathrm{~mL})$. The organic layer was separated, washed successively with a saturated solution of $\mathrm{NaHCO}_{3}$, water and dried over $\mathrm{MgSO}_{4}$. The solvent was evaporated under reduced pressure to afford the crude products $10 \mathrm{a}-\mathbf{i}$.

Procedure C. Cycliacylations using PTSA catalyst. A stirred mixture of acid 3a-i (0.5 g) and PTSA (5.0 g) was heated on an oil bath and kept at the required temperature for a certain time as shown in Tables $1 \& 2$. Afterwards, the flask was cooled to room temperature and the reaction products basified by addition of $\mathrm{NaHCO}_{3}$ solution ( $40 \mathrm{~mL}, 30 \%)$. The residue was extracted with ether $(3 \times 20 \mathrm{~mL})$ and the combined organic phases were washed with water, dried over anhydrous $\mathrm{Na}_{2} \mathrm{SO}_{4}$, and the solvent was evaporated in vacuo to give the crude products $10 \mathbf{a}-\mathbf{i}$.

13,14-Dihydro-5H-benzo[5,6][1,4]thiazocino[3,2-h]quinolin-14(12H)-one (11a). Yellow crystals, $\mathrm{mp} 135-7{ }^{\circ} \mathrm{C}$ (from ethanol); IR (KBr, v, cm$\left.{ }^{-1}\right): 3450,3030,2980,1740,1580,1465,1440,1373,1260,1133,1075,748 ;{ }^{1} \mathrm{H}$ NMR $\left(400 \mathrm{MHz}_{2} \mathrm{CDCl}_{3}, \mathrm{ppm}\right), \delta 4.77\left(2 \mathrm{H}, \mathrm{s}, \mathrm{CH}_{2}\right), 7.03(1 \mathrm{H}, \mathrm{dd}, J 1.2$ and $1.6 \mathrm{~Hz}), 7.25-7.32(2 \mathrm{H}, \mathrm{m}), 7.37(1 \mathrm{H}, \mathrm{dd}$, $J 1.6$ and $1.6 \mathrm{~Hz}), 7.46(1 \mathrm{H}, \mathrm{dt}, J 0.8 \mathrm{~Hz}), 7.54(1 \mathrm{H}, \mathrm{dt}, J 8.0$ and $2.0 \mathrm{~Hz}), 7.88(1 \mathrm{H}$, two t, J 2.0, 1.6, $4.2,2.0$ and 1.2 $\mathrm{Hz}), 8.28(1 \mathrm{H}, \mathrm{dd}, J 1.2$ and $1.6 \mathrm{~Hz}), 8.76(1 \mathrm{H}, \mathrm{dd}, J 2.0$ and $1.6 \mathrm{~Hz}), 9.80(1 \mathrm{H}, \mathrm{s}, \mathrm{NH}) ;{ }^{13} \mathrm{C} \mathrm{NMR}(100 \mathrm{MHz}, \mathrm{CDCl}, \delta$, 
ppm): 38.2, 117.2, 117.6, 122.1, 122.2, 122.5, 126.6, 126.8, 127.9, 130.8, 131.5, 134.4, 140.7, 141.1, 144.8, 148.7, 194.4. Anal. Calcd. for $\mathrm{C}_{17} \mathrm{H}_{12} \mathrm{~N}_{2} \mathrm{OS}$ (292); C, 69.86; H, 4.10; N, 9.58; S, 10.95. Found; C, 69.75; H, 4.18; N, 9.64; S, $10.81 \%$.

13,14-Dihydro-5H-pyrido[2',3'-5,6][1,4]thiazocino[3,2-h]quinolin-14(12H)-one (11b). Pale yellow solid, mp $177-9{ }^{\circ} \mathrm{C}$ (from ethanol); IR (KBr, v, cm $\left.{ }^{-1}\right): 3422,3055,2930,1740,1600,1585,1470,1445,1389,1275,1150$, 755; ${ }^{1} \mathrm{H} \mathrm{NMR}\left(400 \mathrm{MHz}, \mathrm{CDCl}_{3}, \mathrm{ppm}\right), \delta 4.80\left(2 \mathrm{H}, \mathrm{s}, \mathrm{CH}_{2}\right), 6.92(1 \mathrm{H}, \mathrm{q}, J 4.8,2.0$ and $5.6 \mathrm{~Hz}), 7.30-7.34(2 \mathrm{H}, \mathrm{m})$, $7.80(1 \mathrm{H}, \mathrm{dd}, J 1.6$ and $2.0 \mathrm{~Hz}), 7.89(1 \mathrm{H}$, two t, J $1.6 \mathrm{~Hz}), 8.13(1 \mathrm{H}, \mathrm{dd}, J 2.0$ and $2.0 \mathrm{~Hz}), 8.28(1 \mathrm{H}, \mathrm{dd}, J 1.6 \mathrm{and}$ $2.0 \mathrm{~Hz}), 8.76(1 \mathrm{H}, \mathrm{dd}, J 2.0$ and $1.6 \mathrm{~Hz}) 10.27(1 \mathrm{H}, \mathrm{s}, \mathrm{NH}) ;{ }^{13} \mathrm{C} \mathrm{NMR}\left(100 \mathrm{MHz}, \mathrm{CDCl}_{3}, \delta, \mathrm{ppm}\right): 38.2,117.2,118.1$, $122.1,123.3,126.6,127.8,127.9,130.8,134.4,140.7,144.8,148.7,150.5,156.9,194.4$. Anal. Calcd. for $\mathrm{C}_{16} \mathrm{H}_{11} \mathrm{~N}_{3} \mathrm{OS}$ (293); C, 65.52; H, 3.75; N, 14.33; S, 10.92. Found; C, 65.66; H, 3.89; N, 14.42; S, $10.78 \%$.

8,13,14-Trihydro-7H-benzo[6,7][1,4]thiazonino[3,2-h]quinolin-13(15H)-one (11c). White crystals; $\mathrm{mp} 95-6{ }^{\circ} \mathrm{C}$ (from benzene); IR (KBr, v, cm-1): 3380, 3010, 2965, 1743, 1580, 1480, 1440, 1395, 1284, 1135, 1077, 752; ${ }^{1} \mathrm{H}$ NMR (400 MHz, CDCl,$p p m), \delta 4.48\left(2 \mathrm{H}, \mathrm{s}, \mathrm{CH}_{2}\right), 4.61\left(2 \mathrm{H}, \mathrm{s}, \mathrm{CH}_{2}\right), 6.88(1 \mathrm{H}, \mathrm{d}, J 8.8 \mathrm{~Hz}), 7.23-7.27(2 \mathrm{H}, \mathrm{m}), 7.36$ $(1 \mathrm{H}, \mathrm{dt}, J 1.6 \mathrm{~Hz}), 7.46(1 \mathrm{H}, \mathrm{dt}, J 6.4$ and $1.2 \mathrm{~Hz}), 7.71(1 \mathrm{H}, \mathrm{two} \mathrm{t}, J 1.6,1.6,6.8$ and $1.6 \mathrm{~Hz}), 7.81(1 \mathrm{H}, \mathrm{dd}, J 1.2 \mathrm{and}$ $1.6 \mathrm{~Hz}), 8.31(1 \mathrm{H}, \mathrm{dd}, J 1.6$ and $1.6 \mathrm{~Hz}), 8.69(1 \mathrm{H}, \mathrm{dd}, J 2.0$ and $1.6 \mathrm{~Hz}), 9.75(1 \mathrm{H}, \mathrm{s}, \mathrm{NH}) ;{ }^{13} \mathrm{C} \mathrm{NMR}\left(100 \mathrm{MHz} \mathrm{CDCl}_{3}\right.$, $\delta, \mathrm{ppm}): 38.2$, 48.6, 117.2, 122.1, 125.8, 126.6, 126.6, 127.0, 127.9, 130.8, 131.4, 132.3, 133.1, 134.4, 140.7, 148.7, 194.4. Anal. Calcd. for $\mathrm{C}_{18} \mathrm{H}_{14} \mathrm{~N}_{2} \mathrm{OS}$ (306); C, 70.58; $\mathrm{H}, 4.57 ; \mathrm{N}, 9.15 ; \mathrm{S}, 10.45$. Found; C, 70.54; $\mathrm{H}, 4.61 ; \mathrm{N}$, $9.31 ;$ S, $10.29 \%$.

6,7,8-Trihydro-13H-benzo[5,6][1,4]thiazonino[3,2- $h$ ]quinolin-8-one (11d). Yellow needles; mp $138-40{ }^{\circ} \mathrm{C}$ (from acetone); IR (KBr) $v_{\max } 3410,3035,2920,1743,1580,1460,1455,1385,1290,1140,1070,760 \mathrm{~cm}^{-1} ;{ }^{1} \mathrm{H} \mathrm{NMR}$ (400 MHz, $\left.\mathrm{CDCl}_{3}, \mathrm{ppm}\right), \delta 2.79\left(2 \mathrm{H}\right.$, app quin, J 2.8, 1.6, 2.0 and $\left.2.4 \mathrm{~Hz}, \mathrm{CH}_{2}\right), 3.75(2 \mathrm{H}$, app quin, J 3.2, $1.2,1.6$ and $\left.2.8 \mathrm{~Hz}, \mathrm{CH}_{2}\right), 7.02(1 \mathrm{H}, \mathrm{dd}, J 1.2$ and $1.2 \mathrm{~Hz}), 7.24-7.38(4 \mathrm{H}, \mathrm{m}), 7.55(1 \mathrm{H}, \mathrm{dt}, J 2.0,1.6$ and $2.0 \mathrm{~Hz}), 7.88(1 \mathrm{H}$, two t, J 1.6, 1.6 and $1.6 \mathrm{~Hz}), 8.09(1 \mathrm{H}, \mathrm{dd}, J 1.6$ and $2.0 \mathrm{~Hz}), 8.75(1 \mathrm{H}, \mathrm{dd}, J 2.0$ and $2.0 \mathrm{~Hz}), 10.28(1 \mathrm{H}, \mathrm{s}, \mathrm{NH}) ;{ }^{13} \mathrm{C}$ $\mathrm{NMR}\left(100 \mathrm{MHz}_{\mathrm{CDCl}}, \delta, \mathrm{ppm}\right): 30.0,44.9,117.2,117.6,122.1,122.2,124.5,126.6,127.9,130.4,130.8,131.5$, 134.4, 140.7, 141.1, 144.8, 148.7, 199.2. Anal. Calcd. for $\mathrm{C}_{18} \mathrm{H}_{14} \mathrm{~N}_{2} \mathrm{OS}$ (306); C, 70.58; H, 4.57; N, 9.15; S, 10.45. Found; C, 70.44; H, 4.62; N, 9.27; S, $10.38 \%$.

13,14-Dihydro-5H-pyrido[2',3'-5,6][1,4]thiazonino[3,2-h]quinolin-15-one (11e). White needles; mp $143-5{ }^{\circ} \mathrm{C}$ (from acetone); IR (KBr) $v_{\max } 3430,3045,2960,1740,1600,1585,1480,1440,1380,1385,1130,744 \mathrm{~cm}^{-1} ;{ }^{1} \mathrm{H}$ NMR (400 MHz, CDCl, $\mathrm{ppm}), \delta 2.80\left(2 \mathrm{H}\right.$, app quin, J 2.4, 2.0, 1.6 and $\left.2.8 \mathrm{~Hz}, \mathrm{CH}_{2}\right), 3.81(2 \mathrm{H}$, app q, J $2.8,2.0$ and $\left.4.0 \mathrm{~Hz}, \mathrm{CH}_{2}\right), 6.90(1 \mathrm{H}, \mathrm{q}, J 5.2,1.6$ and $5.2 \mathrm{~Hz}), 7.29-7.33(2 \mathrm{H}, \mathrm{m}), 7.56(1 \mathrm{H}, \mathrm{dd}, J 2.0$ and $1.6 \mathrm{~Hz}), 7.89(1 \mathrm{H}, \mathrm{two}$ t, J 2.0, 1.6, 2.0 and $1.6 \mathrm{~Hz}), 8.09(1 \mathrm{H}, \mathrm{dd}, J 1.6$ and $1.6 \mathrm{~Hz}), 8.41(1 \mathrm{H}, \mathrm{dd}, J 2.0$ and $2.0 \mathrm{~Hz}), 8.75(1 \mathrm{H}, \mathrm{dd}, J 2.0 \mathrm{and}$ $1.6 \mathrm{~Hz}), 10.32(1 \mathrm{H}, \mathrm{s}, \mathrm{NH}) ;{ }^{13} \mathrm{C} \mathrm{NMR}\left(100 \mathrm{MHz}, \mathrm{CDCl}_{3}, \delta, \mathrm{ppm}\right): 30.07,44.9,117.2,118.1,122.1,123.3,126.6$, $127.8,127.9,130.8,134.4,140.7,144.8,148.7,150.5,156.9,199.2$. Anal. Calcd. for $\mathrm{C}_{17} \mathrm{H}_{13} \mathrm{~N}_{3} \mathrm{OS}$ (307); C, 66.44; H, 4.23; N, 13.68; S, 10.42. Found; C, 66.45; H, 4.36; N, 13.55; S, $10.50 \%$.

8,9,10,15-Tetrahydro-16H-benzo[6,7][1,4]thiazecino[3,2-h]quinolin-10-one (11f). Pale yellow crystals; $\mathrm{mp}$ 174- $6{ }^{\circ} \mathrm{C}$ (from benzene); IR (KBr) $v_{\max } 3370,3015,2975,1745,1600,1590,1470,1440,1381,1276,1132,758$ $\mathrm{cm}^{-1}$; ${ }^{1} \mathrm{H} \mathrm{NMR}\left(400 \mathrm{MHz}, \mathrm{CDCl}_{3}, \mathrm{ppm}\right), \delta 2.87\left(2 \mathrm{H}, \mathrm{t}, J 6.0\right.$ and $\left.5.6 \mathrm{~Hz}, \mathrm{CH}_{2}\right), 3.93\left(2 \mathrm{H}, \mathrm{t}, J 5.2\right.$ and $\left.6.0 \mathrm{~Hz}, \mathrm{CH}_{2}\right), 4.52$ $\left(2 \mathrm{H}, \mathrm{s}, \mathrm{CH}_{2}\right), 6.89(1 \mathrm{H}, \mathrm{d}, J 8.8 \mathrm{~Hz}), 7.24-7.32(3 \mathrm{H}, \mathrm{m}), 7.46(1 \mathrm{H}, \mathrm{app} \mathrm{dt}, J 6.8$ and $1.2 \mathrm{~Hz}), 7.73(1 \mathrm{H}, \mathrm{two} \mathrm{t}, J 2.0,1.6$ and $1.6 \mathrm{~Hz}), 7.83(1 \mathrm{H}, \mathrm{dd}, J 1.2$ and $1.2 \mathrm{~Hz}), 8.28(1 \mathrm{H}, \mathrm{dd}, J 1.6$ and $1.6 \mathrm{~Hz}), 8.72(1 \mathrm{H}, \mathrm{dd}, J 1.6$ and $1.6 \mathrm{~Hz}), 9.95$ $(1 \mathrm{H}, \mathrm{s}, \mathrm{NH}) ;{ }^{13} \mathrm{C}$ NMR $\left(100 \mathrm{MHz}, \mathrm{CDCl}_{3}, \delta, \mathrm{ppm}\right): 30.0,44.9,48.6,117.2,122.1,125.8,126.6,126.6,127.0,127.9$, 130.8, 131.4, 132.3, 133.1, 140.7, 144.8, 148.7, 199.2. Anal. Calcd. for $\mathrm{C}_{19} \mathrm{H}_{16} \mathrm{~N}_{2} \mathrm{OS}(320) ; \mathrm{C}, 71.25 ; \mathrm{H}, 5.00 ; \mathrm{N}$, $8.75 ;$ S, 10.00. Found; C, 71.33; H, 4.86; N, 8.78; S, $9.87 \%$.

2,3,4,5-Tetrahydro-10H-benzo[5,6][1,4]thiazecino[3,2-h]quinolin-5-one (11g). Yellow needles; mp $138-40{ }^{\circ} \mathrm{C}$ (from acetone); IR (KBr) $v_{\max } 3374,3072,2952,1740,1580,1496,1440,1365,1290,1137,1095,759 \mathrm{~cm}^{-1} ;{ }^{1} \mathrm{H}$ 
NMR (400 MHz, $\left.\mathrm{CDCl}_{3}, \mathrm{ppm}\right), \delta 1.93\left(2 \mathrm{H}\right.$, quin, J 4.4, 4.4, 4.4 and $\left.4.8 \mathrm{~Hz}, \mathrm{CH}_{2}\right), 2.52(2 \mathrm{H}$, app q, J 7.6, 4.8 and 4.4 $\left.\mathrm{Hz}, \mathrm{CH}_{2}\right), 3.62\left(2 \mathrm{H}\right.$, app q, J 4.4, 2.0 and $\left.2.4 \mathrm{~Hz}, \mathrm{CH}_{2}\right), 7.18(1 \mathrm{H}, \mathrm{d}, J 9.2 \mathrm{~Hz}), 7.28-7.40(4 \mathrm{H}, \mathrm{m}), 7.62(1 \mathrm{H}, \mathrm{dt}, J 1.6$, 1.6, 4.2 and $1.6 \mathrm{~Hz}), 7.88(1 \mathrm{H}$, two t, J 1.6, 1.6, 1,6 and $1.6 \mathrm{~Hz}), 8.10(1 \mathrm{H}, \mathrm{dd}, J 1.2$ and $1.2 \mathrm{~Hz}), 8.76(1 \mathrm{H}, \mathrm{dd}, J 2.0$ and $2.0 \mathrm{~Hz}), 10.21(1 \mathrm{H}, \mathrm{s}, \mathrm{NH}) ;{ }^{13} \mathrm{C} \mathrm{NMR}\left(100 \mathrm{MHz}^{\mathrm{CDCl}} \mathrm{C}, \delta, \mathrm{ppm}\right): 26.0,33.2,38.7,117.2,117.6,122.1,122.2$, 124.5, 126.6, 127.9, 130.4, 130.8, 131.5, 134.4, 140.7, 141.1, 144.8, 148.7, 204.3. Anal. Calcd. for $\mathrm{C}_{19} \mathrm{H}_{16} \mathrm{~N}_{2} \mathrm{OS}$ (320); C, 71.25; H, 5.00; N, 8.75; S, 10.00. Found; C, 71.22; H, 5.14; N, 8.64; S, $10.05 \%$.

2,3,4,5-Tetrahydro-10H-pyrido[2',3'-5,6][1,4]thiazecino[3,2-h]quinolin-5-one (11h). White crystals; mp 150-2 ${ }^{\circ} \mathrm{C}$ (from acetone); IR (KBr) $v_{\max } 3366,3045,2930,1745,1595,1485,1440,1430,1374,1280,1174,760 \mathrm{~cm}^{-1} ;{ }^{1} \mathrm{H}$ NMR (400 MHz, CDCl, $\mathrm{ppm}), \delta 1.89\left(2 \mathrm{H}, \mathrm{m}, \mathrm{CH}_{2}\right), 2.54\left(2 \mathrm{H}, \mathrm{app} \mathrm{q}, J 6.8,5.2\right.$ and $\left.4.4 \mathrm{~Hz}, \mathrm{CH}_{2}\right), 3.50(2 \mathrm{H}$, app quin, J 2.4, 2.0, 2.4 and $\left.2.0 \mathrm{~Hz}, \mathrm{CH}_{2}\right), 6.91(1 \mathrm{H}, \mathrm{q}, J$ 5.2, 2.4 and $4.8 \mathrm{~Hz}), 7.24(1 \mathrm{H}, \mathrm{d}, J 9.2 \mathrm{~Hz}), 7.30(1 \mathrm{H}, \mathrm{q}, J 4.8,3.6$ and $4.8 \mathrm{~Hz}), 7.80(1 \mathrm{H}, \mathrm{dd}, J 2.0,5.2$ and $2.0 \mathrm{~Hz}), 7.89(1 \mathrm{H}$, two t, $J 1.6,1.6$ and $1.6 \mathrm{~Hz}), 8.11(1 \mathrm{H}, \mathrm{dd}, J 1.6 \mathrm{and} 1.2 \mathrm{~Hz})$, $8.39(1 \mathrm{H}, \mathrm{dd}, J 2.0$ and $2.0 \mathrm{~Hz}), 8.76(1 \mathrm{H}, \mathrm{dd}, J 2.0$ and $2.0 \mathrm{~Hz}), 9.83(1 \mathrm{H}, \mathrm{s}, \mathrm{NH}) ;{ }^{3} \mathrm{C} \mathrm{NMR}\left(100 \mathrm{MHz}^{\mathrm{C} C D C l}, \delta\right.$, ppm): 26.0, 33.2, 38.7, 117.2, 118.1, 122.1, 123.3, 126.6, 127.8, 127.9, 130.8, 134.4, 140.7, 144.8, 148.7, 150.5, 156.9, 199.2. Anal. Calcd. for $\mathrm{C}_{18} \mathrm{H}_{15} \mathrm{~N}_{3} \mathrm{OS}$ (321); C, 67.28; $\mathrm{H}, 4.67 ; \mathrm{N}, 13.08 ; \mathrm{S}, 9.96$. Found; C, 67.41; $\mathrm{H}, 4.54 ; \mathrm{N}$, $13.14 ;$ S, $10.04 \%$.

8,9,10,11,16-Pentaahydro-17H-benzo[6,7][1,4]thiazacyclododecano[3,2-h]quinolin-11-one (11i). White needles; $\mathrm{mp} 132-4^{\circ} \mathrm{C}$ (from acetone); IR (KBr) $v_{\max } 3430,3080,2950,1737,1584,1485,1440,1435,1375,1290$, 1075, $747 \mathrm{~cm}^{-1} ;{ }^{1} \mathrm{H}$ NMR (400 MHz, $\left.\mathrm{CDCl}_{3}, \mathrm{ppm}\right), \delta 1.87\left(2 \mathrm{H}, \mathrm{sp}, J 5.6,4.4,5.2\right.$ and $\left.4.8 \mathrm{~Hz}, \mathrm{CH}_{2}\right), 2.60(2 \mathrm{H}, \mathrm{t}, J 4.4$, 3.2 and $\left.1.6 \mathrm{~Hz}, \mathrm{CH}_{2}\right), 3.64\left(2 \mathrm{H}, \mathrm{t}, J 5.2\right.$ and $\left.5.6 \mathrm{~Hz}, \mathrm{CH}_{2}\right), 4.78\left(2 \mathrm{H}, \mathrm{s}, \mathrm{CH}_{2}\right), 7.07(1 \mathrm{H}, \mathrm{d}, J 8.8 \mathrm{~Hz}), 7.17(1 \mathrm{H}, \mathrm{dd}, J 1.6$ and $1.2 \mathrm{~Hz}), 7.23(1 \mathrm{H}, \mathrm{q}, J 4.8,3.6$ and $4.8 \mathrm{~Hz}), 7.35(1 \mathrm{H}, \mathrm{dt}, J 1.3,6.4$ and $1.3 \mathrm{~Hz}), 7.46(1 \mathrm{H}, \mathrm{dt}, J 1.6,6.4,1.2$ and $8.0 \mathrm{~Hz}), 7.73(1 \mathrm{H}$, two t, J 1.6, 1.6, 1.3, 1.6 and $1.6 \mathrm{~Hz}), 7.83(1 \mathrm{H}, \mathrm{dd}, J 1.2,6.4$ and $1.6 \mathrm{~Hz}), 8.29(1 \mathrm{H}, \mathrm{dd}, J 1.6 \mathrm{and}$ $1.2 \mathrm{~Hz}), 8.72(1 \mathrm{H}, \mathrm{dd}, J 1.6$ and $1.6 \mathrm{~Hz}), 10.15(1 \mathrm{H}, \mathrm{s}, \mathrm{NH}) ;{ }^{13} \mathrm{C} \mathrm{NMR}\left(100 \mathrm{MHz}, \mathrm{CDCl}_{3}, \delta, \mathrm{ppm}\right): 26.0,33.2,38.7$, 48.6, 117.2, 122.1, 125.8, 126.6, 126.6, 127.0, 127.9, 130.8, 131.4, 132.3, 133.1, 134.4, 140.7, 148.7, 206.8. Anal. Calcd. for $\mathrm{C}_{20} \mathrm{H}_{18} \mathrm{~N}_{2} \mathrm{OS}$ (334); C, 71.85; H, 5.38; N, 8.38; S, 9.58. Found; C, 72.02; H, 5.28; N, 8.44; S, $9.46 \%$.

\section{Acknowledgements}

The author is grateful to Chemistry department, Faculty of science Assiut University, Assiut, Egypt for financial support and for providing research facilities received while performing and writing this work.

\section{References}

1. Fattorusso, E; Taglialatela-Scafati, O., Modern Alkaloid Wiley-VCH, Verlag: Weinheim, 2008.

2. Wang, L.; Zhang, P.; Zhang, X.; Zhang, Y.; Li, Y.; Wang,Y., Eur. J. Med. Chem. 2009, 44, 2815-2821. https://doi.org/10.1016/i.ejmech.2008.12.021

3. Margolis, B. J.; Swidorski, J. J.; Rogers, B. N., J. Org. Chem. 2003, 68, 644-647. https://doi.org/10.1021/jo026546g

4. Grunewald, G. L.; Dahanukar, V. H.; Ching, P., Criscione, K. R. J. Med. Chem. 1996, 39, 3539-46. https://doi.org/10.1021/jm9508292

5. Torres, R.; Mittal, D.; Kennedy, R. Psychosomatics 2001, 42, 347-349. https://doi.org/10.1176/appi.psy.42.4.347 
6. Chiesi, M.; Schwaller, R.; Eichenberger. K. Biochem.Pharmacol. 1988, 37, 4399-4403. https://doi.org/10.1016/0006-2952(88)90623-5

7. Arya, K.; Dandia, A. Bioorganic and Medicinal Chem. Letters 2008, 28, 114-119. https://doi.org/10.1016/i.bmcl.2007.11.002

8. Richard, C. A.; Philip, A. R.; Hansjorg, U., J. Med. Chem. 1978, 21, 838-841. https://doi.org/10.1021/jm00206a027

9. Bariwal , J. B.; Upadhyay, K. D.; Manvar, A.T.; Trivedi, J. C.; Singh, J. S.; Jain, K. S.; Shah, A.K. Eur. J. Med. Chem. 2008, 43, 2279-2290.

https://doi.org/10.1016/i.ejmech.2008.05.035

10. Elks, J.; Ganellin, C. R. Dictionary of Drugs, Chapmann and Hall:London, 1990, pp 867. https://doi.org/10.1007/978-1-4757-2085-3

11. Morton, G. C.; Salvino, J. M.; Labaudinie`re, R. F.; Herpin, T. F. Tetrahedron Lett. 2000, 41, 3029-3033. https://doi.org/10.1016/S0040-4039(00)00341-5

12. Steiner, G.; Franke, A.; Hädicke, E.; Lenke, D.; Teschendorf, H. -J.; Hofmann, H. -P.; Kreiskott, H.; Worstmann, W. J. J. Med. Chem. 1986, 29, 1877-88.

https://doi.org/10.1021/jm00160a015

13. Reddy, J. R.; Ashok, D.; Sharma, P. N. Indian J. Chem B 1993, 32, 404-406.

14. Kugita, H.; Inoue, H.; Ikezaki, M. Jap. Pat. 1971, 46016749; Chem. Abstr. 1971, 75, 63848.

15. Geyer, H. M.; Watzman, N.; Buckley, J. P. J. Pharmacol. Sci. 1970, 59, 964-968. https://doi.org/10.1002/ips.2600590709

16. Incerti, M.; Acquotti, D.; Sandor, P.; Vicini, P. Tetrahederon,2009, 65, 7487-7490. https://doi.org/10.1016/i.tet.2009.07.003

17. Shcherbakova, I., In Comprehensive Heterocyclic Chemistry III, Elsevier: Oxford, Eds. Katritzky, A. R.; Ramsden, C. A.; Scriven, E. F. V.; Taylor, R. J. K., 2008; Vol. 14, pp 255-300.

18. Lévai, A.; Jekő, J.; Gondos, T.; Simon, A.; Tóth, G. J. Heterocycl. Chem. 2007, 44, 1453-1457. https://doi.org/10.1002/jhet.5570440633

19. Stephens, W. D.; Field, L. J. Org. Chem. 1959, 24, 1576-1579. https://doi.org/10.1021/jo01092a610

20. Prakash, O.; Kumar, A.; Sadana, A.; Prakash, R.; Singh, S. P.; Claramunt, R. M.; Sanz, D.; Alkorta, I.; Elguero, J. Tetrahedron 2005, 61, 6642-6651.

https://doi.org/10.1016/i.tet.2005.03.035

21. Levai, A.; Jeko, J. Arkivoc 2008,(xvii), 234-240.

http://dx.doi.org/10.3998/ark.5550190.0009.h22

22. Fu R.; Xu X.; Dang Q. J. Org. Chem. 2005, 70, 10810-10816. https://doi.org/10.1021/jo051873k

23. Calvo L. A., Gonzalez-Ortega A., Marcos R., Perez R. M. and Sanudo M. C. Tetrahedron 2008, 64, 36913700.

https://doi.org/10.1016/i.tet.2008.02.027

24. Yale, H.; Sowinski, F.; Spitzmiller, E. J. Het. Chem. 1972, 9, 899-909.

https://doi.org/10.1002/jhet.5570090426

25. Bates, D. K.; Li, X.; Jog, P. V. J. Org. Chem. 2004, 69, 2750 -2754.

https://doi.org/10.1021/jo035692z

26. Sashida ,H.; Tsuchiya, T. Heterocycles 1984, 22, 1303-1306.

https://doi.org/10.3987/R-1984-06-1303 
27. Manhas, M. S.; Amin, S. G.; Bose, A. K. Heterocycles 1976, 5, 669-699.

https://doi.org/10.3987/S-1976-01-0669

28. Lu, S. M.; Alper, H., J. Am. Chem. Soc. 2005, 127, 14776-14784. https://doi.org/10.1021/ja053650h

29. van Otterlo, W. A. L.; Morgans, G. L.; Khanye, S. D.; Aderibigbe, B. A. A.; Michael, J. P.; Billing, D. G., Tetrahedron Lett. 2004, 45, 9171-9175.

https://doi.org/10.1016/i.tetlet.2004.10.108

30. Pei, Y.; Lilly, M. J.; Owen, D. J.; D’Souza, L. J.; Tang, X.-Q.; Yu, J.; Nazarbaghi, R.; Hunter, A.; Anderson, C. M.; Glasco, S.; Ede, N. J.; James, I. W.; Maitra, U.; Chandrasekaran, S.; Moos, W. H.; Ghosh, S. S. J. Org. Chem. 2003, 68, 92-98.

https://doi.org/10.1021/jo020446t

31. Mukherjee, C.; Biehl, E. Heterocycles 2004, 63, 2309-2318.

https://doi.org/10.3987/COM-04-10169

32. Federsel, H. J.; Glassare, G.; Högström, K.; Wiestal, J.; Zinko, B.; Odman, C. J. Org. Chem. 1995, 60, 25972606.

https://doi.org/10.1021/jo00113a044

33. Abd El-Aal, H. A. K.; Khalaf, A. A. Aust. J. Chem. 2016, 69, 652-661.

https://doi.org/10.1071/CH15526

34. Abd El-Aal, H. A. K.; Khalaf, A. A. Arkivoc 2013, iv, 306-322.

http://dx.doi.org/10.3998/ark.5550190.p008.163

35. Roberts, R. M.; Khalaf, A. A. Friedel-Crafts Chemistry: A Century of Discovery, Marcel Dekker: New York, 1984.

36. McKay, A. F.; Tarlton, E. J.; Petri, S. I.; Steyermark, P. R.; Mosely, M. A. J. Am. Chem. Soc. 1958, 80, 15101517.

https://doi.org/10.1021/ja01539a058

37. Moorthy, J. N.; Senapati, K.; Parida, K. N.; Jhulki, S.; Sooraj, K.; Nair, N. N. J. Org. Chem. 2011, 76, 9593-9601.

https://doi.org/10.1021/jo201491q

38. Johnson, S. W.; Woroch, L. E.; Buell, G. B. J. Am. Chem. Soc. 1949, 71, 1901-1905.

https://doi.org/10.1021/ja01174a001 